\title{
The Riemann Solution for the Injection of Steam and Nitrogen in a Porous Medium
}

\author{
Wanderson Lambert • Dan Marchesin • \\ Johannes Bruining
}

Received: 4 November 2008 / Accepted: 17 May 2009 / Published online: 9 June 2009

(C) Springer Science+Business Media B.V. 2009

\begin{abstract}
We solve the model for the flow of nitrogen, vapor, and water in a porous medium, neglecting compressibility, heat conductivity, and capillary effects. Our choice of injection conditions is determined by the application to clean up polluted sites. We study all mathematical structures, such as rarefaction, shock waves, and their bifurcations; we also develop a systematic method to find fundamental solutions for thermal compositional flows in porous media. In addition, we unexpectedly find a rarefaction evaporation wave which has not been previously reported in any other study.
\end{abstract}

Keywords Steam injection - Riemann problem - Multiphase flow $\cdot$ Hyperbolic systems . Bifurcation

\section{Introduction}

In this study, we are interested in capturing the waves and their bifurcations in a problem of vapor and nitrogen injection in a porous medium that initially was partially saturated with water. We present the model for the flow based on mass balance and energy conservation equations as in Bruining and Marchesin (2006). An application of our study can be in cleaning sites polluted with oleic components. The advantage of adding nitrogen is that it reduces

W. Lambert $(\bowtie)$

Instituto Nacional de Matemática Pura e Aplicada, Estrada Dona Castorina 110,

Rio de Janeiro, RJ 22460-320, Brazil

e-mail: lambert@fluid.impa.br

D. Marchesin

Instituto de Matemática Pura e Aplicada, Rio de Janeiro, Brazil

e-mail: marchesi@impa.br

J. Bruining

TUDelf - Delft University of Technology, 2628 CN Delft, P.O.Box 5048, Delft, The Netherlands e-mail: j.bruining@tudelft.nl 
the temperature and can therefore be expected to leave a less severe ecological footprint of the cleaning procedure.

The models for the three possible phase configurations, i.e., only single phase water, single phase gas, or two phase water and gas subject to local thermodynamic equilibrium are given by systems of three coupled equations of the type:

$$
\frac{\partial}{\partial t} G+\frac{\partial}{\partial x} u F=0
$$

supplemented by appropriate thermodynamical constraints between variables, such as the law of Clausius-Clapeyron. Due to the thermodynamic constraints, we never need to define more than two dependent variables, e.g., $V_{1}, V_{2}$. The variables $V_{1}, V_{2}$ are called the primary variables. Indeed $\left(V_{1}, V_{2}\right)$ is a subset of the following variables: gas saturation $s_{g}$, vapor composition $\psi_{g w}$, and temperature $T$; $\left(V_{1}, V_{2}\right)$ represents the unknowns in each phase configuration; $G:=G\left(V_{1}, V_{2}\right)=\left(G_{1}, G_{2}, G_{3}\right)\left(V_{1}, V_{2}\right)$ and $F:=F\left(V_{1}, V_{2}\right)=\left(F_{1}, F_{2}, F_{3}\right)\left(V_{1}, V_{2}\right)$ are the accumulation vector and the flux vector, respectively; $u$ is a total velocity. In Lambert and Marchesin (2009), we propose a general formalism for solving this class of equations; see also Lambert and Marchesin (2008) for further details.

Systems of conservation equations of type (1) have the feature that the volumetric flow rate $u$ does not appear in the accumulation vector, rather it appears isolated in the flux vector, so that there is an infinite speed mode associated to $u$. In these systems, the total volume is not conserved, so the dependent variable $u$ is not constant. An important result in our theory is that we can obtain the variable $u$ in terms of primary variables, which are unknowns of system (1); we call this variable $(u)$ "secondary variable". In the standard theory of fractional flow, this speed is constant and it is determined from the boundary conditions only. The feature of variable $u$ has appeared in previous studies of several authors, such as Dumoré et al. (1984); however, here we propose a general fractional flow theory to deal with this variable $u$. We combine a set of approximations in the physical model that greatly simplifies the analysis.

In this article, we extend the ideas presented in Bruining and Marchesin (2006) in several ways. First, we consider all different phase configurations where chemical species can exist under thermodynamic equilibrium. We solve the Riemann problem for states lying in the following pair of phase configurations: the left state $L$ is in the spg and the right state $R$ is in the $s p l$, which was not considered in Bruining and Marchesin (2006). We study shocks between different phase configurations, obtaining condensation shocks, which occur between regions containing vapor and regions containing a mixture of water and nitrogen with vapor. In Bruining and Marchesin (2006), only a condensation shock was found in the phase where liquid water, vapor water, and nitrogen coexist under thermodynamic equilibrium.

Here, the methodology developed allows us to investigate the existence of rarefaction waves; we observe an evaporation rarefaction wave, which occurs in regions containing a mixture of water and nitrogen with vapor. We present an example of the Riemann solution for data in two different phase configurations and also analyze the bifurcations in these solutions. This model is an example of a general theory developed for compositional models with two chemical species and two phases that can be applied for different thermal flows; see Lambert and Marchesin (2009) for more mathematical details.

In Sect.2, we present the model that describes the injection of vapor and nitrogen in a one-dimensional horizontal porous rock initially filled with liquid water. In Sect.3, we obtain the fundamental waves (shock and rarefaction) for the Riemann solution in the three phase configurations under thermodynamical equilibrium: $s p g$, where there is water vapor and nitrogen, Sect. 3.2; $s p l$, composed only by liquid water, Sect. 3.3; $t p$, formed by liquid water, water vapor, and nitrogen, Sect. 3.4, where we obtain the condensation shock and the 
newly found evaporation rarefaction wave. In Sect.4, we present the Riemann solution for the problem describing the injection of vapor and nitrogen in the spg configuration into a porous medium filled with water. The conclusions are given in Sect. 5 .

Equations numbered from (33) to (41) are in Appendix A; those from (42) to (59) are in Appendix B.

\section{The Model}

Compositional models (1) in porous media are widely studied in Petroleum Engineering, see Lake (1989). They describe flows where mass transfer of chemicals between phases and possibly temperature changes, need to be tracked. In Lambert et al. (2005b), we have studied the injection of vapor and water in several proportions into a porous medium containing vapor (gaseous $\mathrm{H}_{2} \mathrm{O}$ ), water (liquid $\mathrm{H}_{2} \mathrm{O}$ ), or a mixture. Here, we consider the one-dimensional horizontal flow resulting the injection of vapor and nitrogen in a porous rock cylinder, where we disregard gravity effects and heat conductivity. The rock has constant porosity $\varphi$ and absolute permeability $k$; this flow was considered first in Bruining and Marchesin (2006). We assume that the fluids are incompressible and that the pressure variations along the cylinder are so small that they do not affect the physical properties of the fluids. The effect of diffusive terms (related to capillary pressure, heat conductivity, etc.) is to widen the heat condensation front as well as other shocks, while the convergence of the characteristics tries to sharpen the front. The balance of these effects yields the front width, which is typically a few tenth of centimeters; on the other hand, the distance between injection and production wells is of the order of hundred $\mathrm{m}$. Thus, this width is negligible, and we can set it to zero to simplify our analysis. Darcy's law for multiphase flows relates the pressure gradient with its seepage speed:

$$
u_{w}=-\frac{k k_{r w}}{\mu_{w}} \frac{\partial p}{\partial x}, \quad u_{g}=-\frac{k k_{r g}}{\mu_{g}} \frac{\partial p}{\partial x} .
$$

The water and gas relative permeability functions $k_{r w}\left(s_{w}\right)$ and $k_{r g}\left(s_{g}\right)$ are considered to be functions of their respective saturations (see Appendix Eq. 41); $\mu_{w}$ and $\mu_{g}$ are the viscosities of the liquid and gaseous phases. The "fractional flows" for the liquid and gaseous phase are saturation-dependent functions defined by:

$$
f_{w}=\frac{k_{r w} / \mu_{w}}{k_{r w} / \mu_{w}+k_{r g} / \mu_{g}}, \quad f_{g}=\frac{k_{r g} / \mu_{g}}{k_{r w} / \mu_{w}+k_{r g} / \mu_{g}} .
$$

The saturations $s_{w}$ and $s_{g}$ add to 1. By Eq. 3, the same is true for $f_{w}$ and $f_{g}$. Using Darcy's law (2), the definitions (3) yield:

$$
u_{w}=u f_{w}, \quad u_{g}=u f_{g}, \quad \text { where } \quad u=u_{w}+u_{g} \text { is the total or Darcy velocity. }
$$

We write the equations for the conservation of total mass of water (liquid and gaseous $\mathrm{H}_{2} \mathrm{O}$ ) and nitrogen (gaseous $\mathrm{N}_{2}$ ) as

$$
\begin{gathered}
\frac{\partial}{\partial t} \varphi\left(\rho_{W} s_{w}+\rho_{g w} s_{g}\right)+\frac{\partial}{\partial x} u\left(\rho_{W} f_{w}+\rho_{g w} f_{g}\right)=0 . \\
\frac{\partial}{\partial t} \varphi \rho_{g n} s_{g}+\frac{\partial}{\partial x} u \rho_{g n} f_{g}=0,
\end{gathered}
$$

where $\rho_{W}$ is the liquid water density, which is assumed to be constant, $\rho_{g w}\left(\rho_{g n}\right)$ denotes the concentration of vapor (nitrogen) in the gaseous phase (mass per unit gas volume). 
In order to describe temperature variation, we formulate the energy conservation in terms of enthalpies, see Beek et al. (1999); Bird et al. (2001), as we ignore adiabatic compression and decompression effects. We neglect longitudinal heat conduction and heat losses to the surrounding rock. We assume also that the temperature $T$ in the water, solid, and gas phases is the same. Thus the energy conservation is given by

$$
\frac{\partial}{\partial t} \varphi\left(\hat{H}_{r}+H_{W} s_{w}+H_{g} s_{g}\right)+\frac{\partial}{\partial x} u\left(H_{W} f_{w}+H_{g} f_{g}\right)=0,
$$

here, $\hat{H}_{r}=H_{r} / \varphi$ and $H_{r}, H_{W}$, and $H_{g}$ are the rock, the liquid water, and the gas enthalpies per unit volume; their expressions can be found in Eq. 35.

The unknowns on the system are a subset of $s_{g}, \psi_{g w}, T$, and $u$; the phase of the flow determines which unknowns are used, as will be explained later. The quantity $\psi_{g w}$ represents the composition (molar fraction) of the $\mathrm{H}_{2} \mathrm{O}$ in the gaseous phase; in Sect.3.2, we derive the system of equations where $\psi_{g w}$ is an unknown.

\section{Rarefaction and Shock Waves in Riemann Solutions}

Here, we extend the method of characteristics (or fractional flow theory) for systems of type (1), see Lake (1989); Smoller (1983). Mathematically, fluid injection is modeled by the Riemann-Goursat problem associated to Eqs. 5-7 with initial data:

$$
\begin{cases}\left(s_{g}, \psi_{g w}, T, u\right)_{L} & \text { if } x=0, t>0, \\ \left(s_{g}, \psi_{g w}, T, \cdot\right)_{R} & \text { if } x>0 .\end{cases}
$$

The speed $u_{L}>0$ is specified at the injection point. The (.) (dot) denotes that the total velocity $u_{R}$ is not specified at the right state. In the next sections, we show that $u_{R}$ can be obtained in terms of $u_{L}, s_{g}, \psi_{g w}$, and $T$.

\subsection{Phase Configurations in Thermodynamical Equilibrium}

There are three different phase configurations: a single-phase liquid configuration, $s p l$, in which the pores contain only liquid water; a single-phase gaseous configuration, $s p g$, with vapor and nitrogen; and a two-phase configuration, $t p$, with a mixture of liquid water, gaseous nitrogen, and vapor. In the latter case, the temperature is specified by the concentration of vapor in the gas through Clausius-Clapeyron law, as we will see. We assume that each configuration is in local thermodynamical equilibrium, so we can use Gibbs' phase rule, $f=c-p+2$ where $f$ represents Gibb's number of thermodynamical degrees of freedom, $c$ and $p$ are the number of chemical species and phases, respectively. As in our thermodynamical model the pressure is fixed, the remaining number of thermodynamical degrees of freedom $f_{P}$ (under constant pressure) is rewritten as

$$
f_{P}=c-p+1 \text {. }
$$

Figure $1 \mathrm{~b}$ shows the three-phase configurations in the variables $\left(s_{w}, \psi_{g w}, T\right)$; we choose $s_{w}$ instead of $s_{g}$ for convenience.

For each phase configuration, there are two important groups of variables. There are "primary variables"; in the next sections, we will show that the primary variables are: in the $s p l, T$; in the $t p, s_{g}$, and $T$; in the $s p g, \psi_{g w}$, and $T$. The other variable $u$ is called "secondary variable" because it will be obtained from the primary variables. The remaining variables are trivially obtained in terms of the primary ones. 

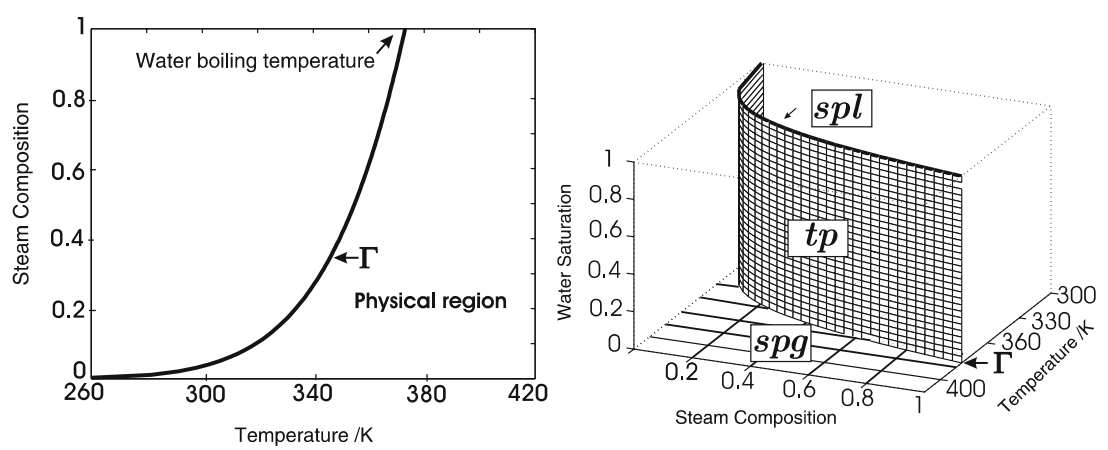

Fig. 1 a left: The physical region for the spg configuration of steam and nitrogen is formed by the pairs $\left(\psi_{g w}, T\right)$ satisfying $\psi_{g w} \leq \Gamma(T)$. The solid graph $\Gamma(T)$ represents the composition of a mixture of nitrogen and steam in equilibrium with liquid water. b right: Phase space for $\left(s_{W}, \psi_{g w}, T\right)$ : single phase gaseous $(s p g)$ configuration (Sect.3.2), single phase liquid ( $s p l)$ configuration (Sect.3.3), and the two phase (tp) configuration (Sect. 3.4)

\subsection{Single-phase Gaseous Configuration—spg}

There are two chemical species $\left(\mathrm{N}_{2}\right.$ and $\left.\mathrm{H}_{2} \mathrm{O}\right)$ and one gaseous phase, i.e., $c=2$ and $p=1$, so the number of thermodynamical degrees of freedom is $f_{P}=2$ : temperature and gas composition. The other unknown is $u$. We define the steam and nitrogen gas compositions, $\psi_{g w}$ and $\psi_{g n}$, as follows, see Bruining and Marchesin (2006); Lambert and Marchesin (2008):

$$
\psi_{g w}=\rho_{g w} / \rho_{g W}(T), \quad \psi_{g n}=\rho_{g n} / \rho_{g N}(T), \quad \text { with } \quad \psi_{g w}+\psi_{g n}=1,
$$

where $\rho_{g W}$ and $\rho_{g N}$ are the densities of pure steam and nitrogen given by Eq. 40 . We assume that in the nitrogen and vapor there are no volume contraction or expansions effects due to mixing so that the volumes of the components are additive: hence Eq. 10c.

Using $\rho_{g w}, \rho_{g n}$ given by Eqs. 10a, b, $f_{w}=s_{w}=0, f_{g}=s_{g}=1$, Eqs. 5-7 become:

$$
\begin{gathered}
\frac{\partial}{\partial t} \varphi \rho_{g W} \psi_{g w}+\frac{\partial}{\partial x} u \rho_{g W} \psi_{g w}=0, \\
\frac{\partial}{\partial t} \varphi \rho_{g N} \psi_{g n}+\frac{\partial}{\partial x} u \rho_{g N} \psi_{g n}=0, \\
\frac{\partial}{\partial t} \varphi\left(\hat{H}_{r}+\rho_{g W} h_{g W} \psi_{g w}+\rho_{g N} h_{g N} \psi_{g n}\right)+\frac{\partial}{\partial x} u\left(\rho_{g W} h_{g W} \psi_{g w}+\rho_{g N} h_{g N} \psi_{g n}\right)=0 ;
\end{gathered}
$$

where the enthalpies per mass unit of pure steam and nitrogen $h_{g W}(T)$ and $h_{g N}(T)$ are used to replace $H_{g}$ (Eq. 35c), and they are given in Eqs. 33-34.

The spg configuration consists of a two-parameter set of triplets $\left(s_{w}=0, \psi_{g w}, T\right)$, satisfying $\psi_{g w} \leq \Gamma(T) \equiv \rho_{g w}(T) / \rho_{g W}(T)$, see Eqs. 39a and 40a, Fig. 1 and Bruining and Marchesin (2006); Lambert and Marchesin (2008). Here, $\left(V_{1}, V_{2}\right)=\left(\psi_{g w}, T\right)$ are the primary variables, and $s_{w}=0, \psi_{g n}=1-\psi_{g w}$.

\subsubsection{Characteristic Speed Analysis}

Assuming that the solution is sufficiently smooth, we differentiate all equations in Eqs. 11-13 with respect to their variables $\left(V_{1}, V_{2}, u\right)$. In the $s p g, V_{1}$ and $V_{2}$ stand for $\psi_{g w}$ and $T$; in the 
$t p, V_{1}$ and $V_{2}$ stand for $s_{g}$ and $T$. For the $s p l$ the system, (5-7) reduces to a scalar transport equation for the unknown $T$. The use of variables $V_{1}$ and $V_{2}$ is appropriate, because the derivation that follows applies to systems of form (1) arising in regions where the solution is smooth. Thus, in the next section, we employ the same derivation for the $t p$ configuration; however, we present only the final results.

The differentiated version of a system of form (1) is written compactly as

$$
\left(B\left(V_{1}, V_{2}\right) \frac{\partial}{\partial t}+A\left(V_{1}, V_{2}, u\right) \frac{\partial}{\partial x}\right)\left(V_{1}, V_{2}, u\right)^{\mathbb{T}}=0,
$$

where the matrices $B$ and $A$ are the derivatives of $G=G\left(V_{1}, V_{2}\right)$ and $u F\left(V_{1}, V_{2}\right)$ with respect to the variables $V_{1}, V_{2}$, and $u$. Since $G$ does not depend on $u$, the last column in the matrix $B$ is zero. The matrices $B$ and $A$ for the spg configuration are:

$$
\begin{gathered}
B=\varphi\left(\begin{array}{ccc}
\rho_{g W} & \rho_{g W}^{\prime} \psi_{g w} & 0 \\
-\rho_{g N} & \rho_{g N}^{\prime} \psi_{g n} & 0 \\
H_{g W}-H_{g N} & \hat{C}_{r}+\psi_{g w} H_{g W}^{\prime}+\psi_{g n} H_{g N}^{\prime} & 0
\end{array}\right) \\
A=\varphi\left(\begin{array}{ccc}
u \rho_{g W} & u \rho_{g W}^{\prime} \psi_{g w} & \rho_{g W} \psi_{g w} \\
-u \rho_{g N} & u \rho_{g N}^{\prime} \psi_{g n} & \rho_{g N} \psi_{g n} \\
u\left(H_{g W}-H_{g N}\right) & u\left(\psi_{g w} H_{g W}^{\prime}+\psi_{g n} H_{g N}^{\prime}\right) & \psi_{g w} H_{g W}+\psi_{g n} H_{g N}
\end{array}\right)
\end{gathered}
$$

We are interested in obtaining the characteristic speeds $\lambda^{i}:=\lambda^{i}\left(V_{1}, V_{2}, u\right)$ and the corresponding characteristic vectors $\boldsymbol{r}^{i}:=\boldsymbol{r}^{i}\left(V_{1}, V_{2}, u\right)$, (here $i$ is the label of each characteristic family). For the system (14), each pair $\lambda^{i}, \boldsymbol{r}^{i}$ is obtained as:

$$
A \boldsymbol{r}^{i}=\lambda^{i} B \boldsymbol{r}^{i} \text { where } \lambda^{i} \text { is obtained by solving } \operatorname{det}\left(A-\lambda^{i} B\right)=0 .
$$

After substitution of $B$ and $A$ given by Eqs. 15 and 16 in Eq. 17b, we notice that $(u-\varphi \lambda)$ is repeated in the first column, so one of eigenpairs is:

$$
\lambda_{c}=u / \varphi, \quad \boldsymbol{r}_{c}=(1,0,0)^{\mathbb{T}},
$$

which corresponds to fluid transport; one can prove that $\nabla \lambda_{c} \cdot \boldsymbol{r}_{c}=0$, so its associated wave is a contact discontinuity. The speed and the temperature are constant, only the composition $\psi_{g w}$ changes, thus we denote this eigenvalue by $\lambda_{c}$ where the subindex c indicates varying composition.

We find the other characteristic speed $\lambda$ and eigenvector in Eq. 17 for $\psi_{g w} \neq 0$ as

$$
\lambda_{T}=\left(1-\hat{C}_{r} T / \mathbb{F}\right) u / \varphi, \quad \boldsymbol{r}_{T}=\left(0, \mathbb{F}, u \hat{C}_{r}\right)^{\mathbb{T}},
$$

where $\mathbb{F}:=\mathbb{F}(T)=\left(\psi_{g w} \rho_{g W}(T) h_{g W}^{\prime}(T)+\psi_{g n} \rho_{g N}(T) h_{g N}^{\prime}(T)+\hat{C}_{r}\right) T$. The first component of vector $\boldsymbol{r}_{T}$ given by Eq. $19 \mathrm{~b}$ is zero, so the associated rarefaction wave has constant composition $\psi_{g w}$. Notice that $\lambda_{T}<\lambda_{c}$ in the physical range of interest; $\rho_{g W}, \rho_{g N}, h_{g W}^{\prime}, h_{g N}^{\prime}$, and $\hat{C}_{r}$ are positive, $\psi_{g w}$ and $\psi_{g n}$ are non-negative, so that $\mathbb{F}(T)$ is always positive.

From the characteristic pair, $\lambda_{T}, \boldsymbol{r}_{T}$, we obtain the thermal rarefaction curves in the $\left(\psi_{g w}, T, u\right)$ space as solutions of the following ODE with increasing $\xi$ :

$$
\left(\frac{d \psi_{g w}}{d \xi}, \frac{d T}{d \xi}, \frac{d u}{d \xi}\right)=\left(0, \mathbb{F}, u \hat{C}_{r}\right) \quad \text { with } \quad \xi=\frac{x}{t}=\lambda_{T}=\left(1-\hat{C}_{r} / \mathbb{F}\right) u / \varphi .
$$

Notice from Eq. 19b that we can solve the ordinary differential equation first for $T$ and $\psi_{g w}$ because the rarefaction curves that parametrize rarefaction waves in $(x, t)$ space do not 

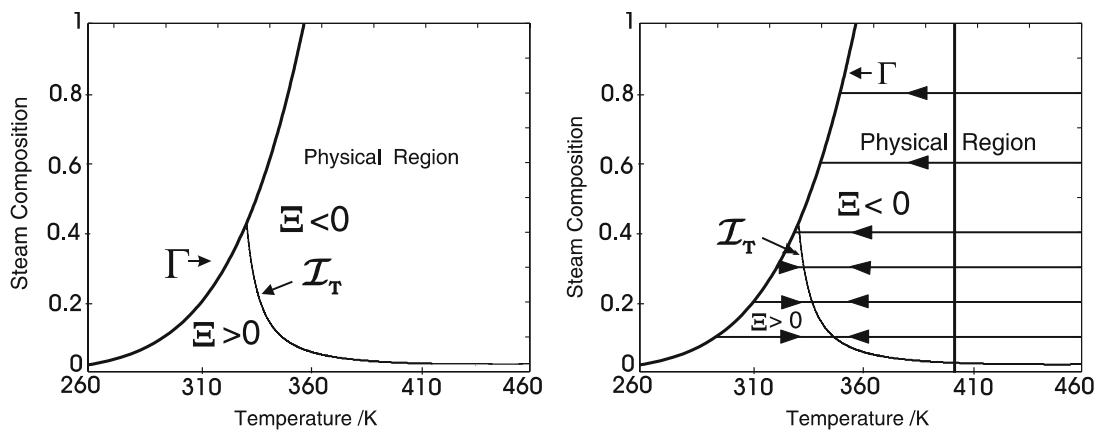

Fig. 2 a Left: The single-phase gaseous physical region $\Gamma$, and inflection locus. b Right: Rarefaction curves. The horizontal rarefaction curves are associated with $\lambda_{T}$; the arrow indicates the direction of increasing speed. The vertical line is a contact discontinuity curve associated with $\lambda_{c}$, in which $\psi_{g w}$ changes, $T$ and $u$ are constant

depend on $u$. Thus, we can substitute $\left(\psi_{g w}, T\right)$ in the equation for the third component of (20a) to obtain $u(\xi)$, which is very simple in the spg:

$$
u(\xi)=u^{-} \exp \left(\int_{\xi^{-}}^{\xi} \hat{C}_{r} d \varsigma\right)=u^{-} \exp \left(\hat{C}_{r}\left(\xi-\xi^{-}\right)\right) .
$$

Here $u^{-}$is the "leftmost" value for $u$ on this rarefaction wave, and $\xi^{-}=\lambda\left(\psi_{g w}^{-}, T^{-}, u^{-}\right)$.

It is a general feature for systems of form (1) that $u$ appears isolated in the eigenvalues and that $u$ appears only in the last component of the eigenvectors, see Lambert and Marchesin (2008).

From a state $\left(\psi_{g w}^{*}, T^{*}\right)$, the states on the rarefaction curve can be drawn with increasing speeds, i.e., satisfying $\nabla_{T} \lambda \cdot \boldsymbol{r}_{T}>0$. Thus, the locus where $\nabla_{T} \lambda \cdot \boldsymbol{r}_{T}=0$ is very important in state space, as it represents the end points of rarefaction curves. For the scalar equation $s_{t}+f(s)_{x}=0$, it corresponds to the state $s$ satisfying $f^{\prime \prime}(s)=0$, hence the name inflection locus. After a lengthly calculation, we obtain

$$
\nabla \lambda_{T} \cdot \boldsymbol{r}_{T}=\left(C_{r} T^{2} \Xi\left(\psi_{g w}, T\right)\right) u / \varphi, \quad \text { where } \quad \Xi=\psi_{g w} \rho_{g W} h_{g W}^{\prime \prime}+\left(1-\psi_{g w}\right) \rho_{g N} h_{g N}^{\prime \prime} .
$$

As $u$ is positive, we need to study the sign of $\Xi$; it vanishes at the gas thermal inflection locus, which is denoted $\mathcal{I}_{T}$. We plot the physical region and $\mathcal{I}_{T}$ in Fig. 2a, showing the signs of $\Xi$. In Fig. 2b, we plot the horizontal rarefaction lines associated with $\lambda_{T}$ and the vertical rarefaction lines associated with $\lambda_{c}$, see Eq. 19.

\subsubsection{Shocks and Contact Discontinuities}

We assume thermodynamical equilibrium in each phase configuration. There exist infinitesimal regions where abrupt changes occur, giving rise to discontinuities. They are shocks in the flow, that satisfy the Rankine-Hugoniot relationships:

$$
v[G]=u^{+} F^{+}-u^{-} F^{-},
$$

where $W^{-}=\left(V_{1}^{-}, V_{2}^{-}, u^{-}\right)$and $W^{+}=\left(V_{1}^{+}, V_{2}^{+}, u^{+}\right)$are the states on the left and the right side of the discontinuity; in the spg configuration, $V_{1}$ and $V_{2}$ stand for $\psi_{g w}$ and $T$. 
The discontinuity speed is $v=v\left(W^{-} ; W^{+}\right)$; the accumulation $G^{-}=G\left(V_{1}^{-}, V_{2}^{-}\right),\left(G^{+}=\right.$ $\left.G\left(V_{1}^{+}, V_{2}^{+}\right)\right)$and flux functions $F^{-}=F\left(V_{1}^{-}, V_{2}^{-}\right)\left(F^{+}=F\left(V_{1}^{+}, V_{2}^{+}\right)\right)$, at the left (right) of the discontinuity; $[G]=G^{+}-G^{-}$. For a fixed $W^{-}$, the set of $W^{+}$states satisfying Eq. 23 defines the Rankine-Hugoniot curve ( $R H$ curve) of $W^{-}$, which is denoted $\mathcal{R H}\left(W^{-}\right)$. We call the shock curve the $W^{+}$that satisfy Eq. 23 and an admissibility criterion, where we assume that the shock speed is decreasing from the (-) state, which is Liu's criterion, see Liu (1974, 1975). The admissibility criterion selects discontinuities that are physical and lead to a unique Riemann solution. We denote the shock state pair between $V^{-}$and $V^{+}$by $\left(V^{-} ; V^{+}\right)$.

Using Eqs. 11-13 in the $R H$ condition, Eq. 23, we obtain:

$$
\begin{gathered}
v \varphi\left(\rho_{g W}^{+} \psi_{g w}^{+}-\rho_{g W}^{-} \psi_{g w}^{-}\right)=u^{+} \rho_{g W}^{+} \psi_{g w}^{+}-u^{-} \rho_{g W}^{-} \psi_{g w}^{-}, \\
v \varphi\left(\rho_{g N}^{+} \psi_{g n}^{+}-\rho_{g N}^{-} \psi_{g n}^{-}\right)=u^{+} \rho_{g N}^{+} \psi_{g n}^{+}-u^{-} \rho_{g N}^{-} \psi_{g n}^{-}, \\
v \varphi\left(H_{r}^{+}+\psi_{g w}^{+} H_{g W}^{+}+\psi_{g n}^{+} H_{g N}^{+}-\left(H_{r}^{-}+\psi_{g w}^{-} H_{g W}^{-}+\psi_{g n}^{-} H_{g N}^{-}\right)\right) \\
=u^{+}\left(\psi_{g w}^{+} H_{g W}^{+}+\psi_{g n}^{+} H_{g N}^{+}\right)-u^{-}\left(\psi_{g w}^{-} H_{g W}^{-}+\psi_{g n}^{-} H_{g N}^{-}\right) .
\end{gathered}
$$

In Appendix B.1, we present a general expression to calculate the $R H$ curve based on equations of type (24-26). Using Eqs. 44, 45, we calculate the shock and Darcy speeds in any problem of type (1). The Darcy speed $u$ usually will not appear in figures because, as proved in Lambert and Marchesin (2008), $u$ can be obtained in terms of $\left(V_{1}, V_{2}\right)$ in each wave (shock or rarefaction). Indeed, from Eq. $23, u^{+}$and $v$ are proportional to $u^{-}$.

One branch of the $R H$ curve for Eqs. 24-26 parametrizes compositional contact discontinuities $C_{\psi}$ with $T$ and $u$ constant and speed $v^{\psi}=u^{-} / \varphi$, see Eq. 18; this type of branch is represented by vertical line in Fig. $2 b$.

The other branch of the $R H$ curve has constant $\psi_{g w}$; it parametrizes thermal shocks with speed $v^{T}$ and Darcy speed $u^{+}$; see the full expressions in Eqs. 46, 47. The thermal shock $S_{T}$ and rarefaction $R_{T}$ are contained in the horizontal lines in Fig. 2 b.

\subsection{Single-phase Liquid Configuration-spl}

There is one chemical species $\left(\mathrm{H}_{2} \mathrm{O}\right)$ and one phase, so there is only $f_{P}=1$ thermodynamical degree of freedom, which is the temperature. Since $s_{w}=1$ and $s_{g}=0$, using Eqs. 3 and 41 we have $f_{w}=1$ and $f_{g}=0$. The liquid is incompressible and composition changes have no volumetric effects, so that the total Darcy velocity $u$ is independent of position. As we assume that rock and water enthalpies depend linearly on temperature, Eqs. 5 and 6 are satisfied trivially and Eq. 7 reduces to (see Lambert et al. (2005a))

$$
\frac{\partial}{\partial t} T+\lambda_{T}^{W} \frac{\partial T}{\partial x}=0, \quad \text { where } \lambda_{T}^{W}=u_{W} \frac{\varphi C_{W}}{\varphi C_{W}+C_{r}},
$$

where we use $u_{W}$ to indicate that the velocity $u$ is spatially constant in the $s p l$ water configuration; here, $C_{W}$ and $C_{r}$ are the water and rock heat capacities. All quantities are given in Appendix A.

Equation 27a is linear in the $s p l$, so that there is a contact discontinuity $C_{T}^{W}$ associated with $\lambda_{T}^{W}$, given by Eq. 27b. It is a cooling discontinuity between states with temperature $T^{-}$ and $T^{+}$. For the corresponding Riemann data, the solution is $T^{-}$if $x / t<\lambda_{T}^{W}$ and $T^{+}$if $x / t>\lambda_{T}^{W}$. 


\subsection{Two-phase Configuration-tp}

There are two chemical species $\left(\mathrm{N}_{2}\right.$ and $\left.\mathrm{H}_{2} \mathrm{O}\right), c=2$, and two phases (liquid water, and gas), $p=2$; so $f_{P}=1$, and we choose the temperature as free thermodynamical variable. As the pressure is given, the boiling temperature of water is specified by the composition of vapor in the gaseous phase. The system is (5-7), and the compositions depend on temperature. When pure vapor is injected, the condensation temperature is the constant $T^{b}=373.15$. We have three variables to be determined: temperature, saturation, and total Darcy velocity. The compositions $\rho_{g w}$ and $\rho_{g n}$ are functions of temperature only, which can be obtained from Eq. 39. The $t p$ state space is composed by the pairs $\left(T, s_{g}\right)$ such that $290 \mathrm{~K} \leq T \leq T^{b}$ and $0 \leq s_{g} \leq 1$. The value $290 \mathrm{~K}$ is chosen arbitrarily, but it is a reasonable temperature for our physical problem.

\subsubsection{Characteristic Speed Analysis}

With a procedure similar to that in Sect.3.2.1, we obtain two eigenpairs in the $t p$. One of them is similar to Buckley-Leverett $(B L)$, and its eigenpair is

$$
\lambda_{s}=\frac{u}{\varphi} \frac{\partial f_{g}}{\partial s_{g}}, \quad \boldsymbol{r}_{s}=(1,0,0)^{\mathbb{T}},
$$

We use the subscript $s$ in $\lambda_{s}$ and $\boldsymbol{r}_{s}$ because $T$ and $u$ are constant, and only $s_{g}$ changes. We denote the rarefaction waves by $R_{B L}$ as vertical lines in Fig. 4a. The wave curves associated with the eigenpair $\lambda_{s}, \boldsymbol{r}_{s}$ are called tie lines in compositional flow theory, see Helfferich (1981); Hirasaki (1981), because on these waves the phase compositions are constant.

The other eigenpair occurs for non-constant $T$ :

$$
\lambda_{e}=\frac{u}{\varphi} \frac{f_{g}-f_{g}^{*}}{s_{g}-s_{g}^{*}} \quad \text { and } \boldsymbol{r}_{e}=\left(-\varpi_{1}, \bar{\gamma}_{1},-u \varpi_{3}\right)^{\mathbb{T}} .
$$

The quantities $f_{g}^{*}, s_{g}^{*}, \varpi_{1}, \bar{\gamma}_{1}$, and $\varpi_{3}$ are given in Eqs. 56-57. We utilize the subscript $e$ to indicate that this eigenpair is associated with an evaporation wave. Evaporation rarefaction waves are denoted by $R_{e}$; the rarefaction wave curves have arrows in Fig. 4a. The arrows indicate the direction of increasing characteristic speed.

In Fig. 3 (as well as 4a), we see that in the region where $\lambda_{s}>\lambda_{e}$, the temperature, gas saturation, and $u$ increase along the rarefaction waves; and in the region where $\lambda_{s}<\lambda_{e}$, the temperature and $u$ increase while the gas saturation decreases along the rarefaction waves.

The coincidence locus between $\lambda_{e}$ and $\lambda_{s}$ is denoted $\mathbb{C}_{s, e}$; it consists of two curves, see Fig. 3a. In Sect. 5.1 of Lambert and Marchesin (2008), we prove that $\partial \lambda_{e} / \partial s_{g}$ vanishes on $\mathbb{C}_{s, e}$, i.e., the coincidence between eigenvalues occurs where $\lambda_{e}$ is stationary. In Fig. 3a, at the lower curve, $\lambda_{e}$ has a minimum and at the higher curve it has a maximum.

In order to obtain the endpoints of evaporation rarefaction curves, we need to study the sign of $\nabla \lambda_{e} \cdot \boldsymbol{r}_{e}$, and since this full expression of this quantity is not crucial to determine the solution, we do not exhibit it in this article; however, we refer to Sect. 5.3 of Lambert and Marchesin (2008) for the expression. The inflection locus $\mathcal{I}_{e}$ consists of the points where $\nabla \lambda_{e} \cdot \boldsymbol{r}_{e}$ vanishes; one can show that they satisfy:

$$
\lambda_{e}=\lambda_{s} \quad \text { or } \quad \frac{f_{g}}{s_{g}}=\frac{\varphi C_{W}}{\varphi C_{W}+C_{r}} .
$$

We define the locus $\mathbb{I}_{e}$ as the states in the $t p$ configuration, which satisfy Eq. 30b, so the inflection locus $\mathcal{I}_{e}$ is formed by union of coincidence locus $\mathbb{C}_{s, e}$ and $\mathbb{I}_{e}$. 

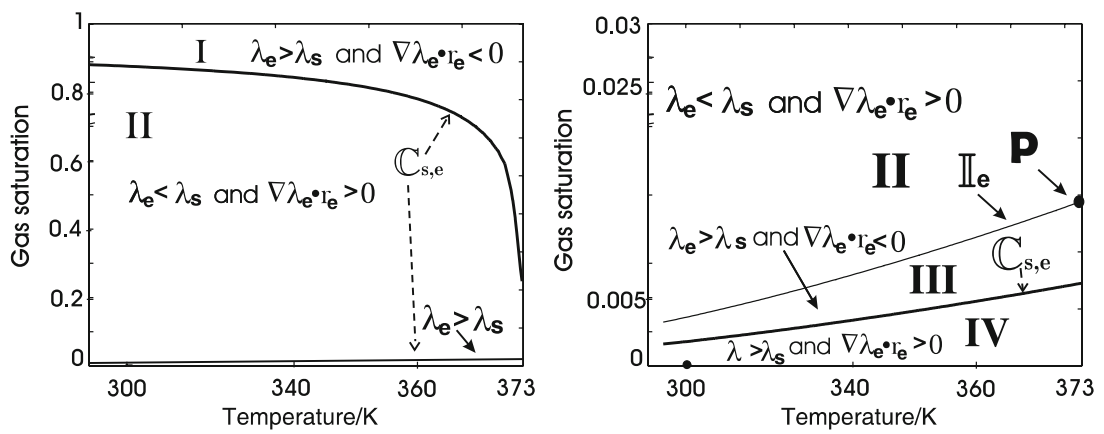

Fig. 3 a Left: Coincidence locus $\mathbb{C}_{s, e}$. Relative sizes of $\lambda_{s}$ and $\lambda_{e}$ in a piece of the $t p$ state space. The almost horizontal coincidence locus $\lambda_{e}=\lambda_{s}$ is not drawn to scale, because it is very close to the axis $s_{g}=0$ and we want to represent the relative size below this locus. b Right: A zoom of the region below the coincidence locus. In both figures, all the curves belong to inflection locus $\mathcal{I}_{e}$, Eq. 22, subdividing the $t p$ configuration in four parts; the point $P$ is the intersection of $\mathbb{I}_{e}$ with the boundary of the $(T, s)$ domain at the water boiling temperature
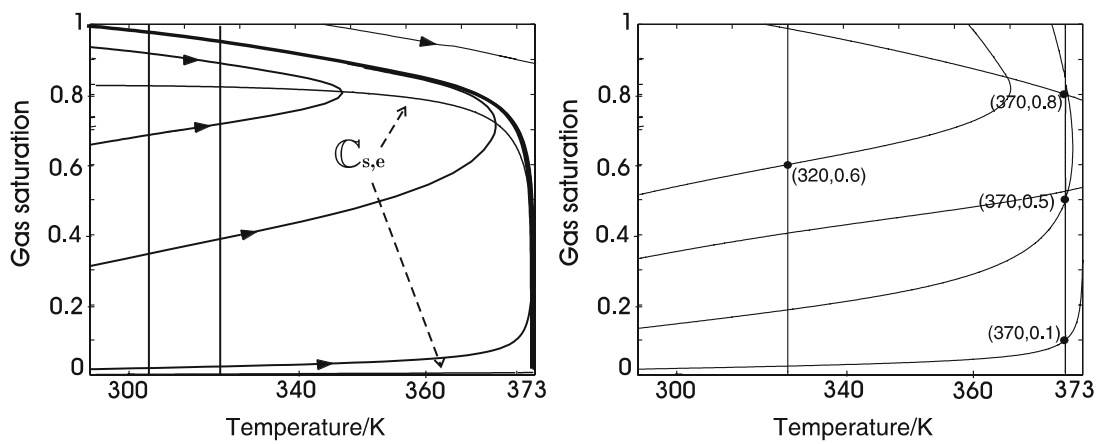

Fig. 4 a Left: Rarefaction curves (with arrows) $R_{e}$ in the $t p$ configuration. The thin curves without arrows are $\mathbb{C}_{s, e}$. The bold curve separating solutions that are invariant under the evolution of evaporation rarefactions is a rarefaction curve reaching the point $\mathbb{P}$ in Fig. 3 b. b Right: The $R H$ curves for $S_{C}$ shocks for the (-) states marked

In Fig. 3a, we draw $\mathbb{C}_{s, e}$; in Fig. 3b, we draw $\mathbb{I}_{e}$ and the lower branch of $\mathbb{C}_{s, e}$; in both figures, we also plot the sign of $\nabla \lambda_{e} \cdot \boldsymbol{r}_{e}$. In Fig. $4 \mathrm{a}$, we draw $R_{e}$ in the $t p$.

\subsubsection{Shock Analysis}

From Eqs. 5-7 and Eq. 23, we obtain the $R H$ relationship, see Appendix B.1.3. The straight line $T=T^{+}$is the isothermal branch of the $R H$ curve; the shock speeds are $v^{B L}=$ $\left[\left(f_{w}\left(s_{w}^{+}, T\right)-f_{w}\left(s_{w}^{-}, T\right)\right) /\left(s_{w}^{+}-s_{w}^{-}\right)\right] u^{-} / \varphi$, and the Darcy speed is constant. These are $B L$ shocks, denoted by $S_{B L}$.

The $R H$ curves for $S_{B L}$ and rarefaction curves $R_{B L}$ associated with $\lambda_{s}$ lie in the same straight lines with constant $T$ in the $t p$ configuration, see Eq. 28 and Fig. $4 \mathrm{~b}$.

The other branch of the $R H$ curve represents non-isothermal condensation shocks, see Bruining and Marchesin (2006). They are denoted by $S_{C}$, see Fig. $4 \mathrm{~b}$. The speed of these shocks is denoted by $v^{C}$. The full expressions for $v^{C}$ and $u^{+}$are given by Eqs. 50, 51 . 


\subsection{Shocks Between Configurations}

The $R H$ relationship for states between distinct configurations is given also by Eq. 23. Notice, however, that the accumulation and flux terms now have different expressions at each shock side.

For the condensation shock $S_{G T}$ between the $s p g$ and the $t p$ configurations, we specify the vapor composition $\psi_{g w}^{-}$, the temperature $T^{-}$, and the Darcy speed $u^{-}$as the left state. For the right state, we specify the temperature $T^{+}$; the vapor composition is a function of temperature (see Eq. 10). The $R H$ expression is given in Sect. B.1.4.

There are also condensation shocks connecting the $t p$ and $s p l$ configurations; however, we do not discuss them here because they do not appear in the example of Riemann solution presented in next section. The full expression is given in Sect. 5.4 of Lambert and Marchesin (2008).

\section{The Riemann Solution for the Clean-up Problem}

We show an example of Riemann solution. We consider the injection of a mixture of vapor (gaseous $\mathrm{H}_{2} \mathrm{O}$ ) and nitrogen in the spg configuration into a rock containing water at a temperature $T_{R}<T^{b}$ (in our examples, we fix $T_{R}=300 \mathrm{~K}$ ) in the $s p l$ configuration:

$$
\left\{\begin{array}{l}
(1, \psi, T, u)_{L} \\
(0, \psi(T), T, \cdot)_{R} \text { if } x>0 .
\end{array} \text { if } x=0 \text { (the injection point), with } u_{L}>0,\right.
$$

There are also minute amounts of contaminant $N A P L$ in the rock; the solution of the Riemann problem determines if the $N A P L$ vaporizes to the gaseous phase.

\subsection{Bifurcation Boundaries and Regions in the Riemann Solution}

1. The double contact. It is composed by the states $(-)=\left(V_{1}^{-}, V_{2}^{-}\right)$in the spg and $(+)=\left(V_{1}^{+}, V_{2}^{+}\right)$in the $t p$ satisfying the $R H$ condition, Eq. 23, and the speed equalities (recall Sect. 3.5):

$$
\lambda_{T}(-)=v^{G T}(-;+) \text { and } v^{G T}(-;+)=\lambda_{B L}(+) .
$$

It is formed by (-) states in the $\operatorname{spg}\left(\mathcal{D}_{S P G}\right)$ and $(+)$ in the $t p\left(\mathcal{D}_{T P}\right)$. Notice that the double contact locus is defined by four unknowns and three equations, so it is a curve in $(-),(+)$ space, see Fig. $5 \mathrm{a}$ for $\mathcal{D}_{S P G}$ and $5 \mathrm{~b}$ for $\mathcal{D}_{T P}$. This locus has an important role in the determination of the Riemann solution, see Sect. 5.2 in Lambert and Marchesin (2008).

2. The curve $E_{H L}$. Extension of physical boundary. It is possible to consider any temperature in the spg; however, very low temperatures lack physical sense. As we consider injection temperatures above $300 \mathrm{~K}$, we take the temperature $T=290 \mathrm{~K}$ as a lower "physical boundary" in the $t p$ configuration.

The curve $E_{H L}$ is formed by the states $V^{-}=\left(\psi_{g w}^{-}, T^{-}\right)$in the spg connected through a shock $S_{G T}$ to $V^{+}=\left(s_{g}^{+}, T^{+}=290 \mathrm{~K}\right)$ in the $t p$ satisfying Eq. 32a, see Fig. 5a. This curve is important because it isolates the states $V^{-}$in $s p g$, such that $S_{G T}$ between $V^{-}$ in $\operatorname{spg}$ reaches states $V^{+}$in $t p$ below $290 \mathrm{~K}$, which we want to exclude.

3. The curve $\mathcal{E}$. This curve is formed by the states $V^{-}=\left(\psi_{g w}^{-}, T^{-}\right)$in the spg connected through a shock $S_{G T}$ to any $V^{+}=\left(s_{g}^{+}, T^{+}=300 K\right)$ in the $t p$ satisfying Eq. $32 \mathrm{~b}$, see Fig. 5a. There is a bifurcation in the Riemann solution structure at $\mathcal{E}$. 

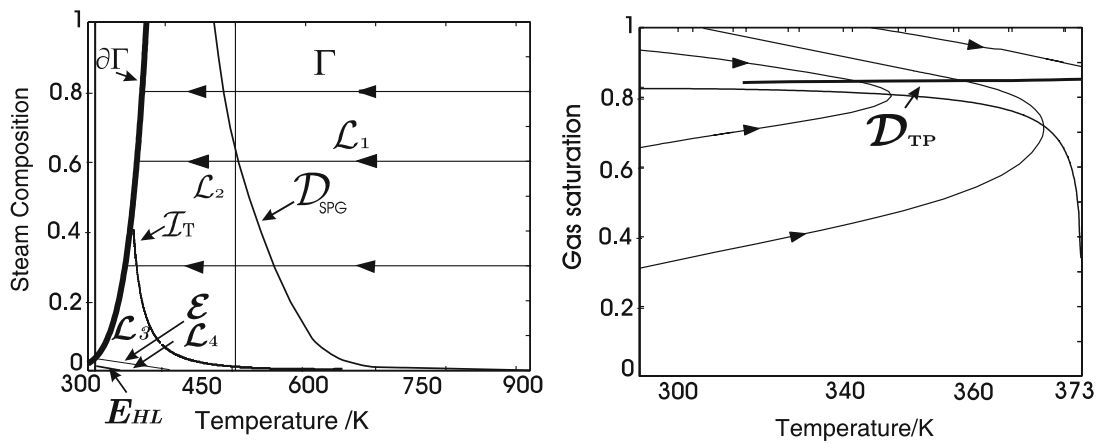

Fig. 5 a Left: The (-) projection of the double contact in the $s p g$, denoted by $\mathcal{D}_{S P G}$, defined in Sect. 4.1.1. The curves $E_{H L}$ and $\mathcal{E}$ are described in Sect.4.1.2 and 4.1.3. The horizontal lines represent the rarefaction with the speed increasing, indicated by the arrows. For a state $V_{L}$ in the region $\mathcal{L}_{i}$ for $i=1,2,3$, 4, defined in Sect.4.1.4, the Riemann solution has the same sequence of waves. b Right: The (+) projection of the double contact in the $t p$, denoted $\mathcal{D}_{T P}$, defined in Sect.4.1.1. Notice that this locus lies above the coincidence locus $\mathbb{C}_{s, e}$. The locus $\mathcal{D}_{T P}$ is the (+) projection of the double contact in the $t p$ configuration. It does not reach the physical boundary $T=290 \mathrm{~K}$, because $\mathcal{D}_{T P}$ is asymptotic to $\psi_{g w}=0$ (only nitrogen) when it reaches the spg configuration. The $(+)$ projection $\mathcal{D}_{T P}$ has an end point in the $t p$ configuration in a state in the interior of the $t p$ configuration
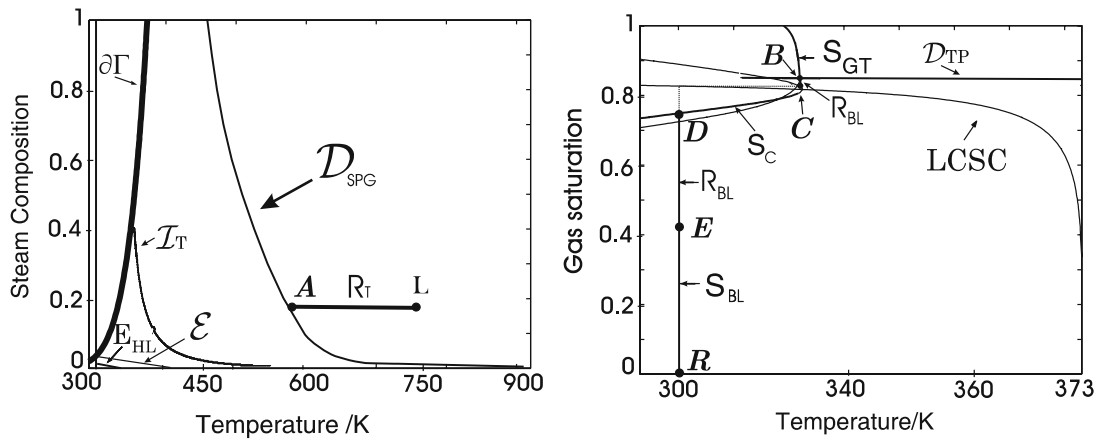

Fig. 6 a Left: Projection of the RS in the spg configuration for $L$ in $\mathcal{L}_{1}$, see Sect. 4.2.1. There is a thermal rarefaction in the spg connecting $R$ to $A$. b Right: Wave sequence in the $t p$ configuration, see Fig. 9a for the solution on the fractional flow curves

4. Regions $\mathcal{L}_{i}$ for $i=1,2,3,4$ in the tp configuration. See Fig. 5a. These regions are separated by the curves $\mathcal{D}_{S P G}, E_{H L}$, and $\mathcal{E}$ just defined, and the thermal inflection curve $\mathcal{I}_{T}$ defined in Sect.3.2.1. The region $\mathcal{L}_{1}$ corresponds to the states on the right of $\mathcal{D}_{S P G}$; the region $\mathcal{L}_{2}$ lies between $\mathcal{D}_{S P G}$ and $\mathcal{I}_{T}$; the region $\mathcal{L}_{3}$ lies between $\mathcal{I}_{T}$ and $\mathcal{E}$; the region $\mathcal{L}_{4}$ is formed by states between $\mathcal{E}$ and $E_{H L}$.

\subsection{The Riemann Solution}

We fix a state given in the second equation in (31) in the $s p l$ configuration. We will obtain the Riemann solution for left states $L$ in each subregion $\mathcal{L}_{i}$ of $s p g, i=1,2,3$, 4, which are shown in Fig. 5a. We shorten notation, and we use $L$ for the left state, $A, B$, etc, for the intermediate states, and $R$ for the right state.

1. Case $\mathcal{L}_{1}$. The solution for $L$ in this region is shown in Figs. 6, 7, and 9a. 

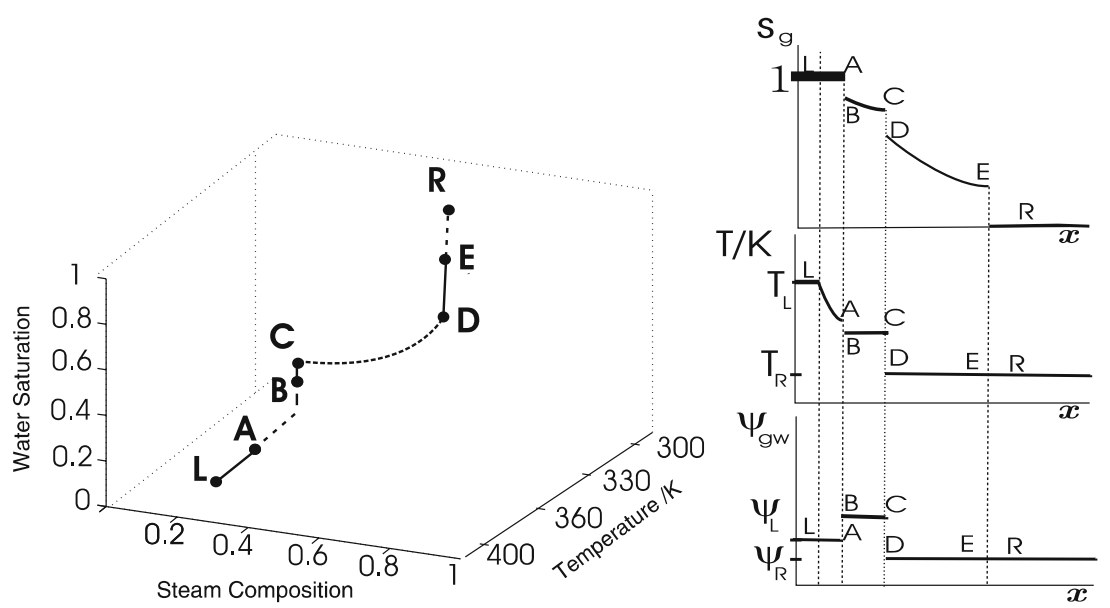

Fig. 7 a Left: Riemann solution in phase space for $L$ in $\mathcal{L}_{1}$, see Sect. 4.2.1 and Figs. 6 and 9a. We omit the $t p$ surface of Fig. 1. b Right: The saturation, temperature, and gas composition profiles for some $t>0$. In a, b the letters $A$ to $E$ indicate intermediate states
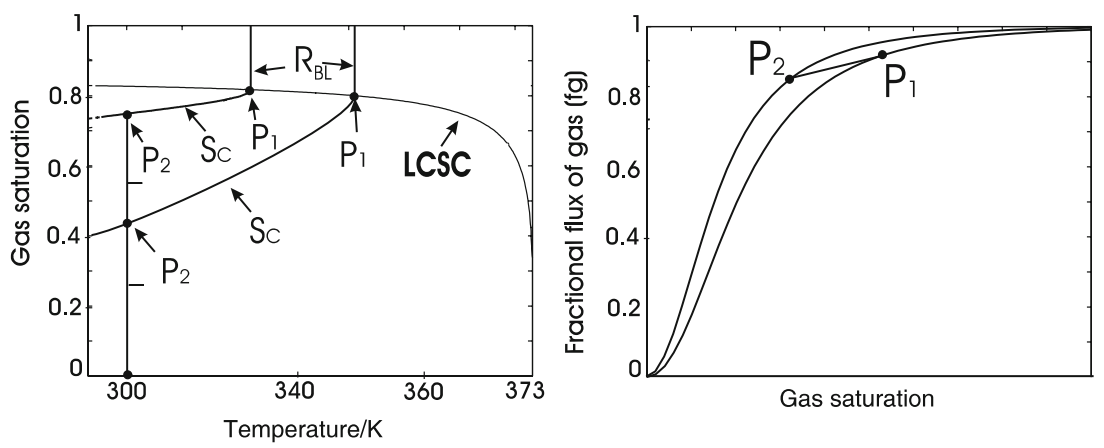

Fig. 8 a left: Two examples of states $P_{1}$ and $P_{2}$. States $P_{1}$ lie on the $R_{B L}$ curve; sweeping all possible $P_{1}$, we describe the $L C S C$, see Sect.4.2.1. Any state $P_{1}$ in $L C S C$ has a corresponding state $P_{2}$ at $T_{R} ;\left(P_{1} ; P_{2}\right)$ is a shock which precedes the last $B L$ wave to $R$. b right: An example of states $P_{1}$ and $P_{2}$. Notice that $P_{1}$ belongs to a $R_{B L}$, with speed given by Eq. 28a. From $P_{1}$ there is a shock $S_{C}$ up to a state $P_{2}$, which satisfies $\lambda_{B L}(P 1)=v^{C}\left(P_{1} ; P_{2}\right)$. Note that the temperature of $P_{1}$ is higher than the temperature of $P_{2}$

See Fig. 6a; notice that for $L$ in $\mathcal{L}_{1}$, we have that $\lambda_{T}(L)<v^{G T}\left(L ; V^{+}\right)$for any $V^{+}$in $S_{G T}$. Here, we use the fact that the wave speeds must increase from $L$ to $R$ states; this statement is called geometrical compatibility of the waves. So from $L$ there is a thermal rarefaction $R_{T}$ up to the double contact $\mathcal{D}_{S P G}$ at a state $A$. From the state $A$, there is a $S_{G T}$ shock between the spg and the $t p$ configurations up to a state $B$, Fig. 6b. This state $B$ belongs both to $\mathcal{D}_{T P}$ and $S_{G T}(A)$ (this means the $S_{G T}$ shock curve starting at the state $A$ ); $B$ satisfies $\lambda_{T}(A)=v^{G T}(A ; B)=\lambda_{B L}(B)$.

From the state $B$ there exists an isothermal rarefaction $R_{B L}$ up to a state $C$, with the same temperature $T_{B}$ of the state $B$. The state $C$ is obtained using the auxiliary "left characteristic condensation shock curve" ( $L C S C$ ), see Fig. 8a. This curve is formed by the auxiliary states $P_{1}$ on $R_{B L}$, such that there is an auxiliary state $P_{2}$ that belongs both to the evaporation shock curve $S_{C}\left(P_{1}\right)$ and to the vertical line $T=T_{R} ; S_{C}$ must satisfy the equality $\lambda_{B L}\left(P_{1}\right)=v^{C}\left(P_{1} ; P_{2}\right)$, see Fig. 8 . Thus, the state $C$ belongs both to the LCSC and to 

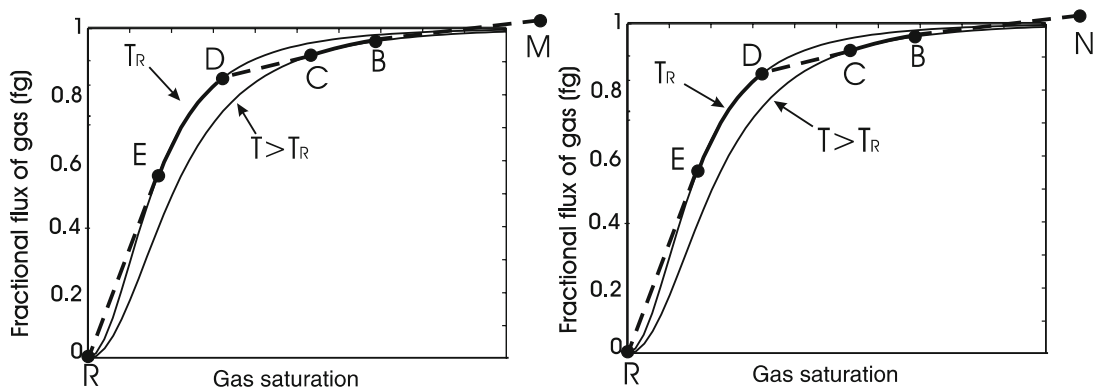

Fig. 9 See Appendix B.3. for the definition of $M$ and $N$. a Left: $L$ in $\mathcal{L}_{1}$. Solution of Sect. 4.2 .1 and Figs. 6 and 7, with fractional flow curves. In both figures, there are two fractional flow curves; one for $T_{R}$ and the other for $T_{B}>T_{R}$. The dashed curves represent shocks and the bold curves represent $B L$ rarefactions. The state $L$ lies in the spg configuration. From $L$ there is $S_{G T}(L ; B)$; the point $M$ is obtained from the RH condition such that the shock is tangent to the fractional flow curve at $B$; from this state, there is a $B L$ rarefaction up to a state $C$ and then $S_{C}(C ; D)$, which is tangent at the state $C$; from the state $D$, there is a $B L$ rarefaction up to a state $E$ and then a $B L$ shock to $R$. b Right: $L$ in $\mathcal{L}_{2}$. Solution of Sect. 4.2.2 and Figs. 10a and 11. Notice that this solution is similar to the previous one
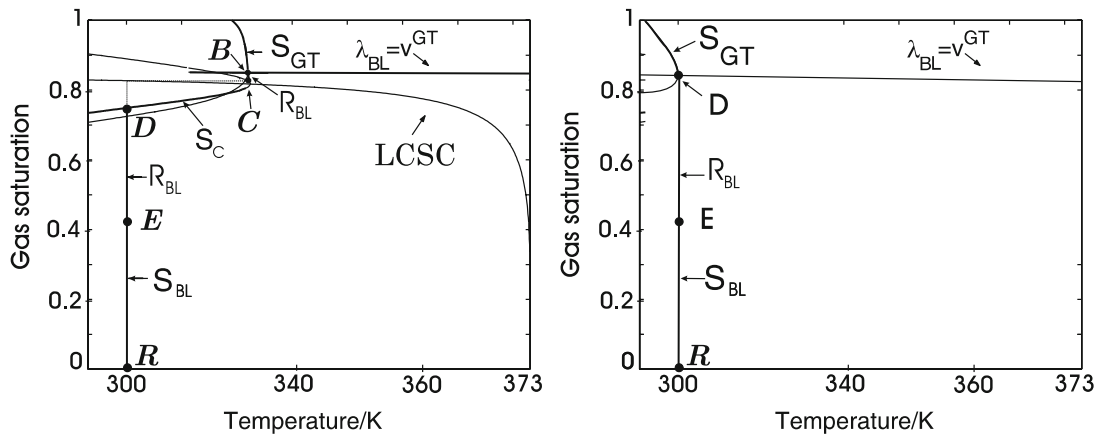

Fig. 10 a Left: Wave sequence in the $t p$ configuration for $L$ in $\mathcal{L}_{2}$ (Sect. 4.2.), see Fig. 9 b for the solution with fractional flow curves. b Right: Wave sequence in the $t p$ configuration for $L$ in $\mathcal{E}$, see Sect. 4.2a and Fig. 12a for the solution on fractional flow curves

the vertical line of $B L$ rarefaction to the right state $R$, see Fig. $6 \mathrm{~b}$. The state $C$ is the basic unknown in the determination of the solution of the Riemann problem. The state $C$ in the $t p$ configuration has two coordinates to be found; one is determined from the left state $L$ and the other from the right state $R$.

The state $D$ belongs both to the $S_{C}$ through the state $C$ and to the vertical line from the right state $R$. From the state $D$ there is an isothermal $B L$ rarefaction up to $E$. The state $E$ is succeded by a $B L$ shock to $R$, with $\lambda_{B L}(E)=v^{B L}(E ; R)$. Condensation of steam occurs in the shocks $S_{G T}(A ; B)$ and $S_{C}(C ; D)$.

2. Case $\mathcal{L}_{2}$. The solution for $L$ in this region is shown in Figs. 9b, 10a and 11 .

The Riemann solution for $L$ can be found as in Case $\mathcal{L}_{1}$, except that $R_{T}$ is absent and from $L$ there is a $S_{G T}$ shock up to a state $B$; this state $B$ belongs both to the curve $\lambda_{B L}(B)=$ $v^{G T}(L ; B)$ and to $S_{G T}(L)$. Notice that $\lambda_{T}(L)>v^{G T}(L ; B)$.

From state $B$, the sequence is the same as in case $\mathcal{L}_{1}$, described in Sect. 4.2.1. 

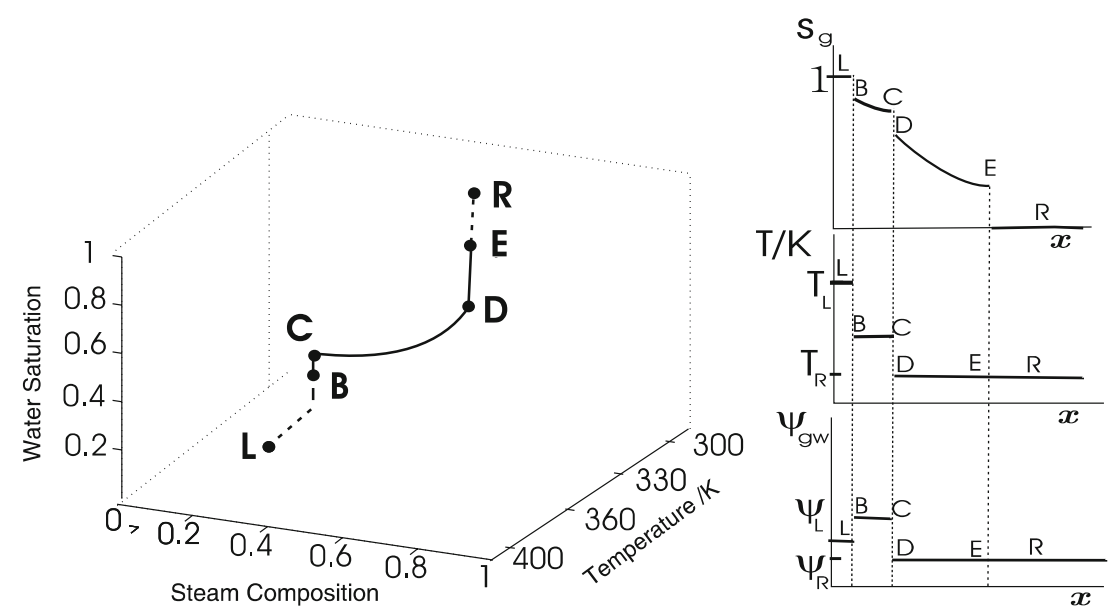

Fig. 11 a $L$ eft: Riemann solution for $L$ in $\mathcal{L}_{2}$, see Sect. 4.2.2 and Figs. 9b and 10a. b Right: The saturation, temperature, and gas composition profiles for fixed $t>0$
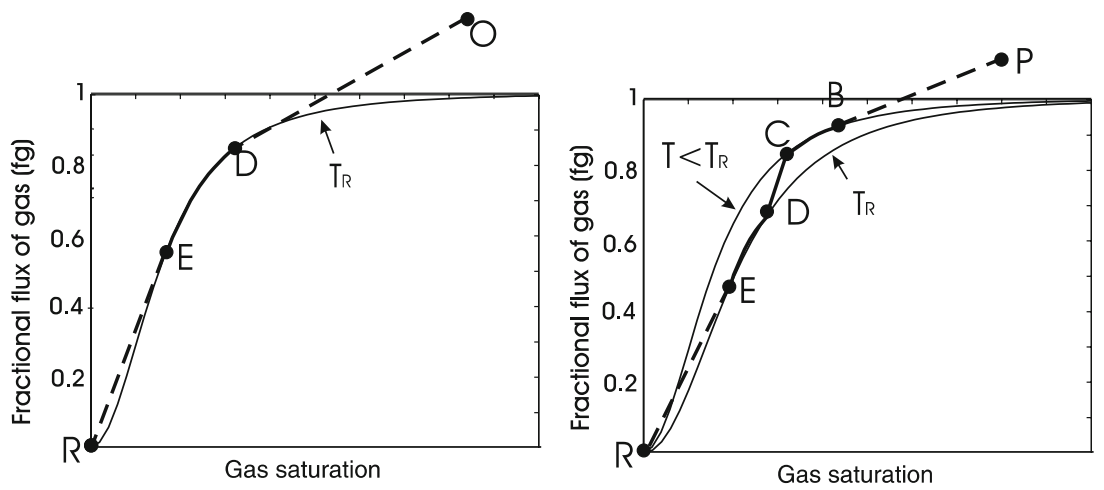

Fig. 12 See Appendix B.3 for the definition of $O$ and $P$. a $L$ eft: $L$ in $\mathcal{E}$. Solution of Sect. 4.2.2a and Figs. 10b and 13 with fractional flow curves for $T_{R}$. The dashed curves represent shocks and the bold curves represent BL rarefactions. From $L$ there is $S_{G T}(L ; D)$ the point $O$ is the point obtained from the RH condition such that the shock is tangent to the fractional flow curve at the state $D$; there is a $B L$ rarefaction up to a state $E$ and then $B L$ shock to $R$. b Right: $L$ in $\mathcal{L}_{3}$. Solution of Sect.4.2.3 and Figs. 14 and 15. Here, there are two fractional flow curves; one for $T_{R}$ and the other for $T_{B}<T_{R}$. From $L$ there is $S_{G T}(L ; B)$; the point $O$ is obtained from the RH condition such that the shock is tangent to the state $B$; from $B$ there is a rarefaction up to a state $C$; from $C$ there is a $R_{e}$ connecting fractional flow at $T_{D}$ and $T_{R}$ in a state $D$; from $D$ there is a $B L$ rarefaction to a state $E$ and then a $B L$ shock to $R$

2.a Case $\mathcal{E}$. The solution is shown in Figs. 10b, 12a, and 13. From $L$ there is a $S_{G T}$ shock between the spg and the $t p$ configurations up to a state $D$, which belongs both to the curve $\lambda_{B L}(D)=v^{G T}(L ; D)$ and to $S_{G T}(L)$, with $\lambda_{T}(L)>v^{G T}(L ; D)$.

Since the temperatures of the state $D$ and of the right state are equal, there is now only a sequence of isothermal $B L$ waves. From the state $D$ there is an isothermal rarefaction up to a state $E$, with $\lambda_{B L}(E)=v^{B L}(E ; R)$.

3. Case $\mathcal{L}_{3}$. The solution for $L$ in this region is shown in Figs. 12b, 14, and 15. 

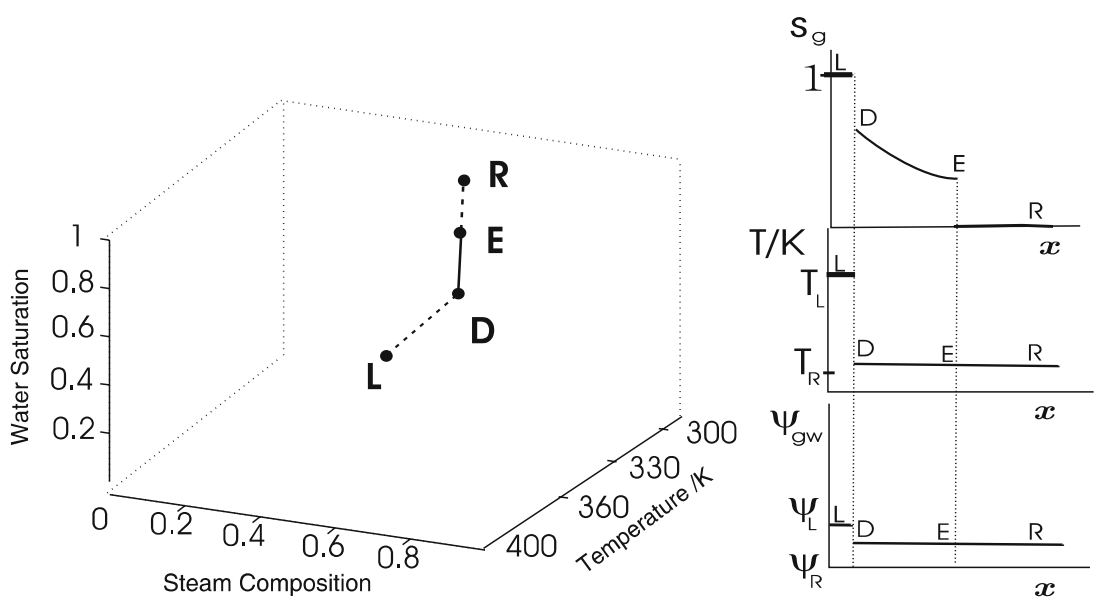

Fig. 13 a Left: Riemann solution in phase space for $L$ in $\mathcal{E}$, see Sect. 4.2.2a and Figs. 10b and 12a. b Right: Saturation, temperature, and gas composition profiles for fixed $t>0$
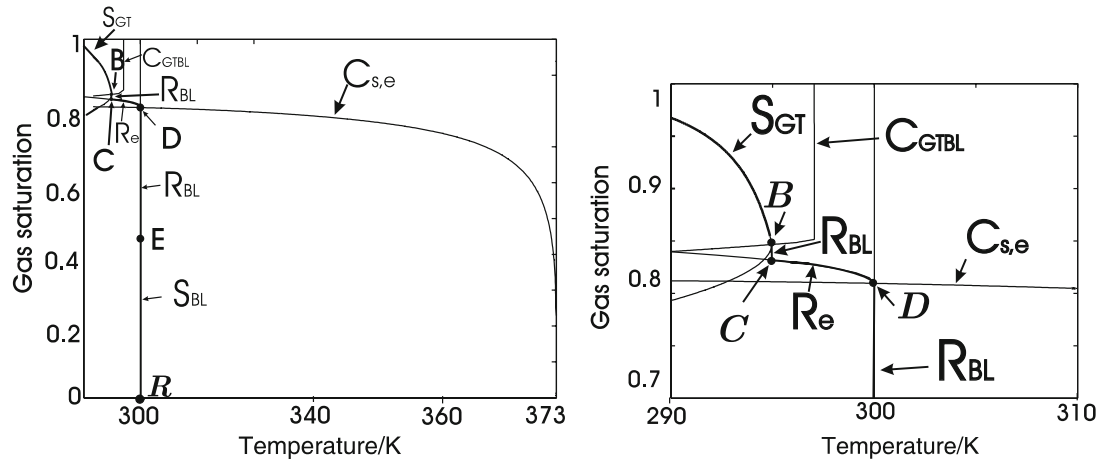

Fig. 14 a $L e f t$ : Wave sequence in $t p$ configuration, $L$ in $\mathcal{L}_{3}$. Notice the existence of a evaporation rarefaction $R_{e}$, Sect. 4.2.3, see Fig. 12.b. b Right: A zoom around the state $B$

From $L$ there is a $S_{G T}$ shock between the spg and the tp configurations up to a state $B$, which belongs both to the curve $\lambda_{B L}(B)=v^{G T}(L ; B)$ and the $S_{G T}(L)$, with $\lambda_{T}(L)>$ $v^{G T}(L ; B)$.

Notice that at $B$ the temperature is below the temperature of the preceding state $R$. This happens because we inject a mixture with high nitrogen concentration and a small quantity of water, so that the enthalpy of the mixture is very low. From the state $B$ there exists an isothermal rarefaction $R_{B L}$ up to a state $C$, with temperature $T_{B}$. The state $C$ is obtained from the coincidence locus between the $R_{B L}$ rarefaction and the (newly found) evaporation rarefaction that crosses the coincidence locus $\mathbb{C}_{s, e}$. From the state $B$ there is an evaporation rarefaction $R_{e}$ up to a state $D$, obtained from the coincidence locus between $R_{e}$ and $\mathcal{C}_{s, e}$. From the state $D$ there is an isothermal rarefaction up to a state $E$. The state $E$ is obtained so as to satisfy the equality $\lambda_{B L}(E)=v^{B L}(E ; R)$. The solution is summarized in Figs. 14 and 15. 

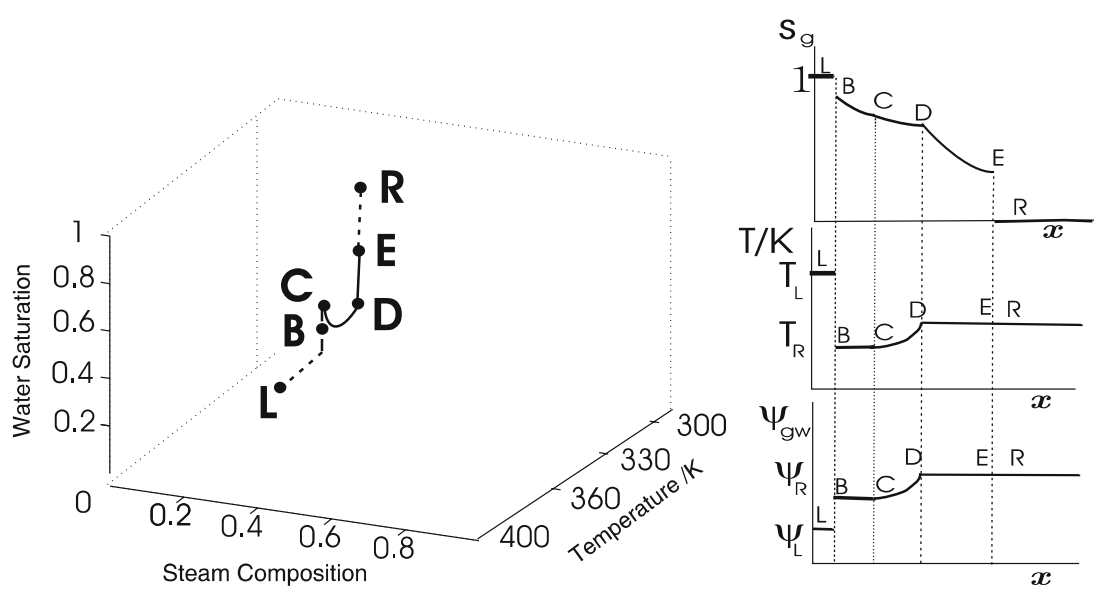

Fig. 15 a Left: Riemann solution in phase space for $L$ in $\mathcal{L}_{3}$, see Sect. 4.2 .3 and Figs. 12b and 14. b Right: The saturation, temperature, and gas composition profiles for some $t>0$

\section{Summary}

We have described the solution of the Riemann problem for the injection of a mixture of nitrogen and vapor into a porous rock filled with water and sketched a systematic theory for the Riemann solution for systems of form (1); these systems encompass compositional problems where the Darcy speed is not constant. The set of solutions depends continuously in the integral sense on the Riemann data. We have generalized the thermal fractional flow theory to deal with variable velocity $u$. This is a step toward obtaining a general method for solving Riemann problems for a wide class of thermal compositional models with phase changes, Lambert (2006).

The Riemann solution agrees with the wave sequence described qualitatively by Davis $(1998,1997)$. A new structure appears in the solution, an evaporation rarefaction $R_{e}$. Practically, this study can be used to find optimal injection strategies to clean up NAPL's from soil with a mixture of nitrogen and vapor. The main advantage of using nitrogen is that operating conditions are below the boiling temperature of water.

\section{Appendix}

\section{A Physical Quantities; Symbols and Values}

The steam enthalpy $h_{g W}[\mathrm{~J} / \mathrm{kg}]$ as a function of temperature is approximated by

$$
\begin{aligned}
h_{g W}(T)= & -2.20269 \times 10^{7}+3.65317 \times 10^{5} T-2.25837 \times 10^{3} T^{2}+7.3742 T^{3} \\
& -1.33437 \times 10^{-2} T^{4}+1.26913 \times 10^{-5} T^{5}-4.9688 \times 10^{-9} T^{6}-\bar{h}_{w} .
\end{aligned}
$$

The nitrogen enthalpy $h_{g N}[\mathrm{~J} / \mathrm{kg}]$ as a function of temperature is approximated by

$$
h_{g N}(T)=975.0 T+0.0935 T^{2}-0.476 \times 10^{-7} T^{3}-\bar{h}_{g N} .
$$


The constants $\bar{h}_{w}$ and $\bar{h}_{g N}$ are chosen so that $h_{w}(T), h_{g N}(T)$ vanish at a reference temperature $\bar{T}=293 \mathrm{~K}$. In the range $[290 \mathrm{~K}, 500 \mathrm{~K}], h_{g W}$ and $h_{g N}$ are almost linear.

The rock enthalpy $H_{r}, \hat{H}_{r}$, water, and gaseous enthalpies per mass unit $H_{W}$ and $H_{g}$ are given by:

$$
H_{r}=C_{r}(T-\bar{T}), \hat{H}_{r}=H_{r} / \varphi, H_{W}=\rho_{W} h_{w} \text { and } H_{g}=\rho_{g w} h_{g W}+\rho_{g n} h_{g N} .
$$

The temperature dependent liquid water viscosity $\mu_{w}[\mathrm{Pas}]$ is approximated by

$$
\begin{aligned}
\mu_{w}= & -0.0123274+\frac{27.1038}{T}-\frac{23527.5}{T^{2}}+\frac{1.01425 \times 10^{7}}{T^{3}}-\frac{2.17342 \times 10^{9}}{T^{4}} \\
& +\frac{1.86935 \times 10^{11}}{T^{5}} .
\end{aligned}
$$

We assume that the viscosity of the gas is independent of the composition.

$$
\mu_{g}=1.8264 \times 10^{-5}\left(T / T^{b}\right)^{0.6} .
$$

The water saturation pressure as a function of temperature is given as

$$
\begin{aligned}
p^{\text {sat }}= & 10^{3}\left(-175.776+2.29272 T-0.0113953 T^{2}+0.000026278 T^{3}\right. \\
& \left.-0.0000000273726 T^{4}+1.13816 \times 10^{-11} T^{5}\right)^{2}
\end{aligned}
$$

The graph of this function looks like a growing parabola.

From the ideal gas law, the corresponding concentrations $\rho_{g w}(T), \rho_{g n}(T)$ are:

$$
\rho_{g w}(T)=M_{W} p^{s a t} /(R T), \quad \rho_{g n}(T)=M_{N}\left(p_{a t}-p^{s a t}\right) /(R T),
$$

where the gas constant $R=8.31[\mathrm{~J} / \mathrm{mol} / \mathrm{K}]$. The pure phase densities are:

$$
\rho_{g W}(T)=M_{W} p_{a t} /(R T), \quad \rho_{g N}(T)=M_{N} p_{a t} /(R T) .
$$

Here, $M_{W}$ and $M_{N}$ are the nitrogen and water molar masses.

The relative permeability functions $k_{r w}$ and $k_{r g}$ are considered to be quadratic functions of their respective reduced saturations. Quadratic functions are often employed for immiscible two-phase flow in porous media as they capture the main behavior of relative permeability functions. More complicated relative permeability functions such as Brooks-Corey could also be used. The form of relative permeability functions for quadratic functions are:

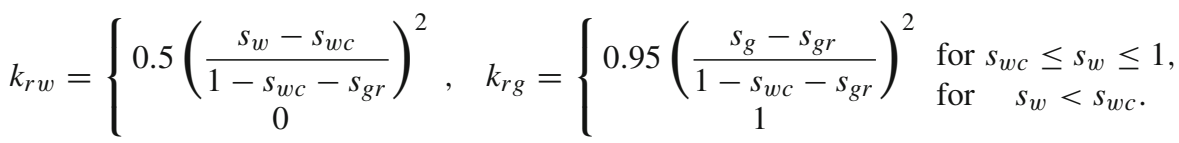


A.1 Summary of physical quantities

Table 1 Summary of physical input parameters and variables

\begin{tabular}{llll}
\hline Physical quantity & Symbol & Value & Unit \\
\hline Water, steam fractional functions & $f_{w}, f_{g}$ & Eq. 3 & {$\left[\mathrm{~m}^{3} / \mathrm{m}^{3}\right]$} \\
Pressure & $p_{a t}$ & $1.0135 \times 10^{5}$ & {$[\mathrm{~Pa}]$} \\
Water Saturation Pressure & $p^{s a t}$ & Eq. 38 & {$[\mathrm{~Pa}]$} \\
Water, steam phase velocity & $u_{w}, u_{g}$ & Eq. 2 & {$\left[\mathrm{~m}^{3} /\left(\mathrm{m}^{2} \mathrm{~s}\right)\right]$} \\
Total Darcy velocity & $u$ & $u_{w}+u_{g}$, Eq. 4 & {$\left[\mathrm{~m}^{3} /\left(\mathrm{m}^{2} \mathrm{~s}\right)\right]$} \\
Effective rock and water heat capacities & $C_{r}$ and $C_{W}$ & $2.029 \times 10^{6}$ and $4.018 \times 10^{6}$. & {$\left[\mathrm{J} /\left(\mathrm{m}^{3} \mathrm{~K}\right)\right]$} \\
Steam and nitrogen enthalpies & $h_{g W}, h_{g N}$ & Eqs. 34a, 34b & {$\left[\mathrm{J} / \mathrm{m}^{3}\right]$} \\
Water enthalpy & $h_{W}$ & $h_{W}=C_{W} T / \rho_{w}$ & {$\left[\mathrm{~J} / \mathrm{m}^{3}\right]$} \\
Rock enthalpy & $H_{r}$ & Eq. 35 & {$\left[\mathrm{J} / \mathrm{m}^{3}\right]$} \\
Water, steam saturations & $s_{w}, s_{g}$ & Dependent variables & {$\left[\mathrm{m}^{3} / \mathrm{m}^{3}\right]$} \\
Connate water saturation & $s_{w c}$ & 0.15 & {$\left[\mathrm{~m}^{3} / \mathrm{m}^{3}\right]$} \\
Temperature & $T$ & Dependent variable & {$[\mathrm{K}]$} \\
Water, steam viscosity & $\mu_{w}, \mu_{g}$ & Eq. 36,37 & {$[\mathrm{~Pa} \mathrm{~s}]$} \\
Steam and nitrogen densities & $\rho_{g w}, \rho_{g n}$ & Eq. $39 \mathrm{a}, 39 \mathrm{~b}$ & {$\left[\mathrm{~kg} / \mathrm{m}^{3}\right]$} \\
Constant water density & $\rho_{W}$ & 998.2 & {$\left[\mathrm{~kg} / \mathrm{m}^{3}\right]$} \\
Steam and nitrogen gas composition & $\psi_{g w}, \psi_{g n}$ & Dependent variables & {$[-]$} \\
Nitrogen and water molar masses & $M_{N}, M_{W}$ & $0.28,0.18$ & {$\left[\mathrm{~kg} / \mathrm{mol}^{3}\right]$} \\
Rock porosity & $\varphi$ & 0.38 & {$\left[\mathrm{~m}^{3} / \mathrm{m}^{3}\right]$} \\
\hline
\end{tabular}

\section{B Analytical Expressions for the Model}

Here, we compile the auxiliary expressions appearing in this article, for further details see Lambert and Marchesin (2008).

B.1 Shock Structures in the $s p l$, or $t p$ Configurations and Between Phase Configurations

1. General theory for shocks. To obtain shocks, by using appropriate $G$ and $F$, we rewrite the system (24-26) in the form:

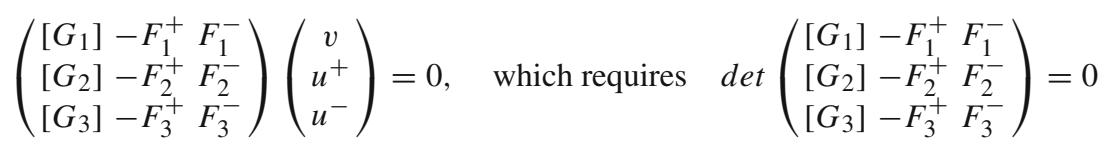

to have a non-trivial solution. Eq. $42 \mathrm{~b}$ yields the implicit expression $\mathcal{H}=0$ with:

$$
\mathcal{H}:=\left[G_{1}\right]\left(F_{3}^{+} F_{2}^{-}-F_{3}^{-} F_{2}^{+}\right)+\left[G_{2}\right]\left(F_{1}^{+} F_{3}^{-}-F_{1}^{-} F_{3}^{+}\right)+\left[G_{3}\right]\left(F_{2}^{+} F_{1}^{-}-F_{2}^{-} F_{1}^{+}\right) .
$$

Since we obtain the RH locus first in the variables $\left(V_{1}^{+}, V_{2}^{+}\right)$by solving $\mathcal{H}=0$ for fixed $\left(V_{1}^{-}, V_{2}^{-}\right)$, it is useful to denote this locus in the variables $V$ by $\mathcal{R H}\left(V_{1}^{-}, V_{2}^{-}\right)$. 
Once $R H\left(V_{1}^{-}, V_{2}^{-}\right)$is determined, we obtain $v$ and $u^{+}$as functions of $\left(V_{1}^{-}, V_{2}^{-}\right),\left(V_{1}^{+}\right.$, $V_{2}^{+}$) and $u^{-}$by solving Eq. 42 a obtaining:

$$
\begin{gathered}
v=u^{-} \frac{F_{2}^{+} F_{1}^{-}-F_{1}^{+} F_{2}^{-}}{F_{1}^{+}\left[G_{2}\right]-F_{2}^{+}\left[G_{1}\right]}=u^{-} \frac{F_{3}^{+} F_{2}^{-}-F_{2}^{+} F_{3}^{-}}{F_{2}^{+}\left[G_{3}\right]-F_{3}^{+}\left[G_{2}^{+}\right]}=u^{-} \frac{F_{1}^{+} F_{3}^{-}-F_{3}^{+} F_{1}^{-}}{F_{3}^{+}\left[G_{1}\right]-F_{1}^{+}\left[G_{3}\right]}, \\
u^{+}=u^{-} \frac{F_{1}^{-}\left[G_{2}\right]-F_{2}^{-}\left[G_{1}\right]}{F_{1}^{+}\left[G_{2}\right]-F_{2}^{+}\left[G_{1}\right]}=u^{-} \frac{F_{2}^{-}\left[G_{3}\right]-F_{3}^{-}\left[G_{2}\right]}{F_{2}^{+}\left[G_{3}\right]-F_{3}^{+}\left[G_{2}\right]}=u^{-} \frac{F_{3}^{-}\left[G_{1}\right]-F_{1}^{-}\left[G_{3}\right]}{F_{3}^{+}\left[G_{1}\right]-F_{1}^{+}\left[G_{3}\right]} .
\end{gathered}
$$

2. Thermal shock in the spg configuration. Using Eqs. 44, 45, after some algebra we obtain the thermal shock speed $v^{T}$ described in Sect.3.2.2:

$$
\begin{aligned}
& v_{T}=\frac{u^{-}}{T^{-}}\left\{\Upsilon\left(T^{-} ; T^{+}\right)\left(T^{+}-T^{-}\right)+T^{+}\right\} \text {and } u^{+}=\frac{u^{-}}{\varphi} \Upsilon\left(T^{-} ; T^{+}\right), \text {where } \\
& \Upsilon=\frac{\psi_{g w}\left(H_{g W}^{+}-H_{g W}^{-}\right)+\left(1-\psi_{g w}\right)\left(H_{g N}^{+}-H_{g N}^{-}\right)}{\hat{H}_{r}^{+}-\hat{H}_{r}^{-}+\psi_{g w}\left(H_{g W}^{+}-H_{g W}^{-}\right)+\left(1-\psi_{g w}\right)\left(H_{g N}^{+}-H_{g N}^{-}\right)} .
\end{aligned}
$$

3. The condensation shock in the tp configuration. Defining $G^{-}=G\left(V^{-}\right), F^{-}=F\left(V^{-}\right)$, $G^{+}=G\left(V^{+}\right)$and $F^{+}=F\left(V^{+}\right)$, where:

$$
\begin{aligned}
& G=\left(\varphi\left(s_{w} \rho_{W}+s_{g} \rho_{g w}\right), \varphi s_{g} \rho_{g n}, H_{r}+\varphi\left(s_{w} H_{w}+s_{g} H_{g}\right)\right)^{\mathbb{T}}, \\
& F=\left(f_{w} \rho_{W}+f_{g} \rho_{g w}, f_{g} \rho_{g n}, \hat{H}_{r}+f_{w} H_{w}+f_{g} H_{g}\right)^{\mathbb{T}},
\end{aligned}
$$

one obtains the $R H$ condition, substituting $G^{-}, \cdots, F^{+}$in Eq. 23. The $R H$ locus is obtained by solving $\mathcal{H}$ given by Eq. 43 with the definitions above. The isothermal branch of the $\mathrm{RH}$ curve is a Buckley-Leverett shock described in Sect.3.4.2. The non-isothermal RH branch is obtained after a lengthly calculation. We fix the left state (-) and the temperature $T^{+}$of the right state, with $T^{+} \neq T^{-}$. Using Eq. 45, we obtain the speed of the condensation shock, denoted by $v^{C}$, as well as the Darcy speed $u^{+}$:

$$
\begin{aligned}
& v^{C}=\frac{u^{-}}{\varphi} \frac{f_{g}^{+} f_{g}^{-}\left(\rho_{g n}^{+}\left(\rho_{g w}^{-}-\rho_{W}\right)-\left(\rho_{g w}^{+}-\rho_{W}\right) \rho_{g n}^{-}\right)-\rho_{W}\left(f_{g}^{+} \rho_{g n}^{+}-f_{g}^{-} \rho_{g n}^{-}\right)}{f_{g}^{+} s_{g}^{-}\left(\rho_{g n}^{+}\left(\rho_{g w}^{-}-\rho_{W}\right)-\left(\rho_{g w}^{+}-\rho_{W}\right) \rho_{g n}^{-}\right)-\rho_{W}\left(s_{g}^{+} \rho_{g n}^{+}-s_{g}^{-} \rho_{g n}^{-}\right)}, \\
& u^{+}=u^{-} \frac{f_{g}^{-} s_{g}^{+}\left(\rho_{g n}^{+}\left(\rho_{g w}^{-}-\rho_{W}\right)-\left(\rho_{g w}^{+}-\rho_{W}\right) \rho_{g n}^{-}\right)-\rho_{W}\left(s_{g}^{+} \rho_{g n}^{+}-s_{g}^{-} \rho_{g n}^{-}\right)}{f_{g}^{+} s_{g}^{-}\left(\rho_{g n}^{+}\left(\rho_{g w}^{-}-\rho_{W}\right)-\left(\rho_{g w}^{+}-\rho_{W}\right) \rho_{g n}^{-}\right)-\rho_{W}\left(s_{g}^{+} \rho_{g n}^{+}-s_{g}^{-} \rho_{g n}^{-}\right)} .
\end{aligned}
$$

4. The condensation shock between the tp the spg configurations. We proceed as above, but remember that $F$ and $G$ have different expressions on the left and right, given by

$$
\begin{aligned}
& G^{-}=\left(\varphi \rho_{g W}^{-} \psi_{g w}^{-}, \varphi \rho_{g N}^{-} \psi_{g n}^{-}, H_{r}^{+}+\varphi\left(\rho_{g W}^{-} h_{g W}^{-} \psi_{g w}^{-}+\rho_{g N}^{-} h_{g N}^{-} \psi_{g n}^{-}\right)\right)^{\mathbb{T}}, \\
& F^{-}=\left(\rho_{g W}^{-} \psi_{g w}^{-}, \rho_{g N}^{-} \psi_{g n}^{-}, \hat{H}_{r}^{+}+\rho_{g W}^{-} h_{g W}^{-} \psi_{g w}^{-}+\rho_{g N}^{-} h_{g N}^{-} \psi_{g n}^{-}\right)^{\mathbb{T}}, \\
& G^{+}=\left(\varphi\left(s_{w}^{+} \rho_{W}+s_{g}^{+} \rho_{g w}^{+}\right), \varphi s_{g}^{+} \rho_{g n}^{+}, H_{r}^{+}+\varphi\left(s_{w}^{+} H_{w}^{+}+s_{g}^{+} H_{g}^{+}\right)\right)^{\mathbb{T}}, \\
& F^{+}=\left(f_{w}^{+} \rho_{W}+f_{g}^{+} \rho_{g w}^{+}, f_{g}^{+} \rho_{g n}^{+}, \hat{H}_{r}^{+}+f_{w}^{+} H_{w}^{+}+f_{g}^{+} H_{g}^{+}\right)^{\mathbb{T}} .
\end{aligned}
$$

One can obtain the $R H$ condition, substituting $G^{-}, F^{-}, G^{+}$and $F^{+}$in Eq. 23. The $R H$ locus is obtained by solving $\mathcal{H}$ given by Eq. 43 with the definitions above; the shock and Darcy speeds $v^{G T}$ and $u^{+}$are obtained by from Eqs. 44,45 . 
B.2 Auxiliary Quantities for Rarefaction Wave in the $t p$

In Sect. 3.4.1, we have obtained the eigenpair $\left(\lambda_{e}, \boldsymbol{r}_{e}\right)$ by using the auxiliary quantities:

$$
\begin{aligned}
& f_{g}^{*}(T)=-\frac{C_{W} \rho_{W}}{\varsigma}, \quad s_{g}^{*}(T)=-\frac{\left(C_{W}+C_{r} / \varphi\right) \rho_{W}}{\varsigma}, \quad \varpi_{3}=\frac{\bar{\gamma}_{1} \bar{\gamma}_{4}\left(\rho_{g w}^{\prime} \rho_{g n}-\rho_{g n}^{\prime} \gamma_{2}\right)}{\rho_{W} \rho_{g n}} \\
& \varpi_{1}=\bar{\gamma}_{4}\left(\frac{\rho_{g n}^{\prime} \rho_{W}-f_{g}\left(\rho_{g w}^{\prime} \rho_{g n}-\rho_{g n}^{\prime} \gamma_{2}\right)}{\rho_{g n} \rho_{W}}\right)+\frac{\partial f_{g}}{\partial T}, \quad \bar{\gamma}_{1}=\frac{\partial f_{g}}{\partial s_{g}}-\frac{f_{g}-f_{g}^{*}}{s_{g}-s_{g}^{*}} .
\end{aligned}
$$

where:

$$
\begin{aligned}
& \varsigma(T)=\left[\left(\gamma_{3}^{\prime}-\left(\rho_{g n}^{\prime} / \rho_{g n}\right) \gamma_{3}\right) \rho_{W}-H_{W}\left(\rho_{g w}^{\prime}-\left(\rho_{g n}^{\prime} / \rho_{g n}\right) \gamma_{2}\right)\right], \quad \bar{\gamma}_{4}=\frac{f_{g}^{*} s_{g}-f_{g} s_{g}^{*}}{s_{g}-s_{g}^{*}}, \\
& \gamma_{2}:=\gamma_{2}(T)=\rho_{g w}-\rho_{W}, \quad \text { and } \quad \gamma_{3}:=\gamma_{3}(T)=H_{g}-H_{W} .
\end{aligned}
$$

B.3 Obtaining the Points $M, N, O$, and $P$ in Figs. 9,12

The points $M, N, O$, and $P$ are auxiliary obtained from the $S_{G T}$. Substituting the the second coordinate of $F^{ \pm}$and $G^{ \pm}$, given by (52-55), in (23), we obtain:

$$
v^{G T} \varphi\left(\rho_{g n}^{+} s_{g}^{+}-\psi_{g n}^{-} \rho_{g N}^{-}\right)=u^{+} \rho_{g n}^{+} f_{g}^{+}-u^{-} \psi_{g n}^{-} \rho_{g N}^{-},
$$

which gives $u^{-}$in terms of $V^{-}, V^{+}, u^{+}$and $v^{G T}$ as

$$
u^{-}=\frac{-v^{G T} \varphi\left(\rho_{g n}^{+} s_{g}^{+}-\psi_{g n}^{-} \rho_{g N}^{-}\right)+u^{+} \rho_{g n}^{+} f_{g}^{+}}{\psi_{g n}^{-} \rho_{g N}^{-}} .
$$

Substituting $u^{-}$in the equation obtained by substituting the first coordinate of $F^{ \pm}$and $G^{ \pm}$, given by (52-55) in (23), we obtain $v^{G T}$ in terms of $V^{-}, V^{+}, u^{+}$:

$$
v^{G T}=\frac{u^{+}}{\varphi} \frac{f_{g}-f_{g}^{\dagger}}{s_{g}-s_{g}^{\dagger}}, \quad \text { where } \quad f_{g}^{\dagger}=s_{g}^{\dagger}=\frac{\rho_{W} \psi_{g n}^{-} \rho_{g N}^{-}}{\left(\rho_{W}-\rho_{g w}^{+}\right) \psi_{g n}^{-} \rho_{g N}^{-}+\psi_{g w}^{-} \rho_{g W}^{-} \rho_{g n}^{+}} .
$$

Given the left state $L$, from the wave sequence, see Sect.4.2.1, we obtain the state $A$ in $\mathcal{D}_{S P G}$. The point $M=\left(s_{g}^{\dagger}, f_{g}^{\dagger}\right)$ is obtained noticing that the shock between $A$ e $B$ has speed equal to the BL characteristic speed, i.e., the shock is tangent to the fractional flow, that satisfies:

$$
\frac{f_{g}-f_{g}^{\dagger}}{s_{g}-s_{g}^{\dagger}}=\frac{\partial f_{g}}{\partial s_{g}}
$$

An alternative form to obtain the state $V^{+}=\left(s_{g}^{+}, T^{+}\right.$) is by solving (43) (using $G^{ \pm}$and $F^{ \pm}$ given by (52-55)) and (63).

The points $N=\left(s_{g}^{\dagger}, f_{g}^{\dagger}\right), O=\left(s_{g}^{\dagger}, f_{g}^{\dagger}\right)$ and $P=\left(s_{g}^{\dagger}, f_{g}^{\dagger}\right)$ are obtained similarly. see Appendix Table 1. 


\section{References}

Beek, W.J., Mutzall, M.K., Van Heuven, J.W.: Transport Phenomena, 2nd edn., 342 pp. Wiley, New York (1999)

Bird, R.B., Stewart, W.E., Lighfoot, E.N.: Transport Phenomena, 2nd edn., 912 pp. Wiley, New York (2001)

Bruining, J., Marchesin, D., Van Duijn, C.J.: Steam injection into water-saturated porous rock. Comput. App. Math. 22(3), 359-395 (2003)

Bruining, J., Marchesin, D.: Nitrogen and steam injection in a porous medium with water. Transp. Porous Media 62(3), 251-281 (2006)

Davis, E.L.: How heat can enhance in-situ soil and aquifer remediation: important chemical properties and guidance on choosing the appropriate technique. EPA/540/S-97/502 (1997)

Davis, E.L.: Steam injection for soil and aquifer remediation. EPA/540/S-97/505 (1998)

Dumoré, J.M., Hagoort, J. Risseeuw, A.S.: An analytical model for one-dimensiona, three-component condensing and vaporizing gas drives, pp. 169-179. SPE, April (1984)

Helfferich, F.G.: Theory of multicomponent, multiphase displacement in porous media. SPEJ 51-62 (1981)

Hirasaki, G.: Application of the theory of multicomponent multiphase displacement to three component, two phase surfactant flooding. SPEJ (1981) 191-204

Lake, L.W.: Enhanced Oil Recovery, 600 pp. Prentice Hall, Englewood Cliffs (1989)

Lambert, W.: Doctoral thesis: Riemann solution of balance systems with phase change for thermal flow in porous media. IMPA. www.preprint.impa.br (2006)

Lambert, W., Marchesin, D.: The Riemann problem for compositional flows in porous media with mass transfer between phases. IMPA. www.preprint.impa.br (2008)

Lambert, W., Marchesin, D.: The Riemann problem for compositional flows in porous media with mass transfer between phases. IMPA, to appear in J. Hyperbolic Differ Equ. (2009)

Lambert, W., Bruining, J., Marchesin, D.: Erratum: steam injection into water-saturated porous rock. Comput. App. Math. 24(3), 1-4 (2005a)

Lambert, W., Marchesin, D., Bruining, J.: The Riemann Solution of the balance equations for steam and water flow in a porous medium. Methods Anal. Appl. 12, 325-348 (2005b)

Liu, T.P.: The Riemann problem for general $2 \times 2$ conservation Laws. Trans. A.M.S. 199, 89-112 (1974)

Liu, T.P.: The Riemann problem for general systems of conservation laws. J. Diff. Equ. 18, 218-234 (1975)

Tsypkin, G.G., Woods, A.W.: Vapour extraction from a water-saturated geothermal reservoir. J. Fluid Mech. 506, 315-330 (2004)

Smoller, J.: Shock Waves and Reaction-Diffusion Equations, 581 pp. Springer-Verlag, Berlin (1983) 